\title{
Free-Surface Effects on Interaction of Multiple Ships Moving at Different Speeds ${ }^{\&}$
}

\author{
Zhi-Ming Yuan a, b, Liang Li ${ }^{a}$ and Ronald W. Yeung c,* \\ aUniversity of Strathclyde, UK \\ bJiangsu University of Science and Technology, China. \\ cUniversity of California at Berkeley, USA \\ *Correspondence author, E-mail: rwyeung@berkeley.edu, Tel.: +1(510) 642-8347
}

\begin{abstract}
Ships often have to pass each other in proximity in harbor area and waterways in dense shipping traffic environment. Hydrodynamic interaction occurs when a ship is overtaking (or being overtaken) or encountering other ships. Such an interactive effect could be magnified in confined waterways, e.g. shallow and narrow rivers. Since Yeung (1978) published his initial work on ship-interaction in shallow water, progress on unsteady interaction among multiple ships has been slow though steady over the following decades. With some exceptions, nearly all the published studies on ship-to-ship problem neglected free-surface effects, and a rigid wall condition has often been applied on the water surface as the boundary condition. When the speed of the ships is low, this assumption is reasonably accurate, as the hydrodynamic interaction is mainly induced by near-field disturbances. However, in many maneuvering operations, the encountering or overtaking speeds are actually moderately high (Froude number $F_{n}>0.2$, where $F_{n} \equiv U / \sqrt{g L}, U$ is ship speed, $g$ the gravitational acceleration and $L$ the ship length), especially when the lateral separation between ships is the order of ship length. Here, the far-field effects arising from ship waves can be important. The hydrodynamic interaction model must take into account of the surface-wave effects.
\end{abstract}

Classical potential-flow formulation is only able to deal with the boundary value problem (BVP) when there is only one speed involved in the free-surface boundary condition. For multiple ships travelling with different speeds, it is not possible to express the free-surface boundary condition by a single velocity potential. Instead, a superposition method can be applied to account for the velocity field induced by each vessel with its own and unique speed. The main objective of the present paper is to propose a rational superposition method to handle the unsteady free-surface boundary condition containing two or more speed terms, and validate its feasibility in predicting the hydrodynamic hydrodynamic behavior in ship encountering. The methodology used in the present paper is a three-dimensional boundary-element method (BEM) based on a Rankine-type (infinite-space) source function, initially introduced in Bai \& Yeung (1974). The numerical simulations are conducted by using an in-house developed multi-body hydrodynamic interaction program "MHydro". Waves generated and forces (or moments) are calculated when ships are encountering or passing each other. Published model-test results are used to validate our calculations and very good agreement has been observed. The numerical results show that free-surface effects need to be taken into account for $F_{n}>0.2$.

\footnotetext{
\& Revised and updated version of Paper (\#109) presented at the 32nd Symposium on Naval Hydrodynamics held at Hamburg, Germany, August 5-10, 2018.
} 
Keywords: Free-surface effect; ship-to-ship problem; hydrodynamic interaction; encountering and overtaking operation; ship maneuvering.

\section{INTRODUCTION}

The interaction between two or more ships involved in encountering or overtaking manoeuvring is a classical hydrodynamic problem. Because of the interaction forces, a ship may deviate from its intended course and collide with the other ships. The interaction effects are aggravated when the ships are manoeuvring in confined waterways, or when the ships are travelling with high speed.

Ship-to-ship problem has been widely studied over the last few decades. No matter which kind of methods are used, at least one or more of the following important assumptions are often adopted to simplify the problem:

1) The fluid is ideal and the viscous effects are neglected.

2) The speed is low and the free-surface effects are negligible ("rigid freesurface" is applicable).

3) The ships are slender.

4) The shedding of cross-flow vorticity is either ignored, or idealized in a manner similar to thin-wing theory.

During1960s-1990s, the slender-body theory has been widely popular to predict the hydrodynamic interaction between multiple ships (Collatz, 1963; Dand, 1975; Kijima and Yasukawa, 1985; Tuck, 1966; Tuck and Newman, 1974; Varyani et al., 1998; Yeung, 1978). All of the assumptions mentioned above were adopted in these studies. These assumptions significantly simplified the mathematical model and led to a high-efficiency numerical calculation method. For conventional ships travelling at relatively low Froude numbers, the numerical calculations based on strip theory showed a fairly good prediction of the sway force and yaw moment on ships during overtaking or meeting operations. To account for the three-dimensional effects and remove the geometrical idealization described above (Assumption 3)), Korsmeyer et al. (1993) adopted a threedimensional panel method, which is applicable to any number of arbitrary shaped bodies in arbitrary motions. Pinkster (2004) extended Korsmeyer's method with implementation of a model to account for the free-surface effects partially. His model was restricted to simulating the effect of a passing ship on a moored ship. Only the low frequency seiche or solitary waves were taken into account, while the more important far-field waves or so-called Kelvin waves were neglected. Therefore, his conclusions on free-surface effects could not cover the general ship-to-ship operations. More recently, the three-dimensional panel method has been more commonly used (Söding and Conrad, 2005; Xiang and Faltinsen, 2010; Xu et al., 2016; Zhou et al., 2012). However, no effort has yet been made to investigate the effects of unsteady free-surface waves on interaction forces. The general conclusion drawn from these earlier studies is that the potential-flow solver could provide a good prediction of interaction forces on ships travelling at relatively low Froude numbers. Benefitted from improving CFD (Computational Fluid Dynamics) technology, the viscous effects on shipto-ship problem have been investigated with various turbulence models (Jin et al., 2016; Sian et al., 2016; Zou and Larsson, 2013). In these studies, the freesurface effects are either neglected (Zou and Larsson, 2013) or treated simply as a steady problem (Jin et al., 2016; Sian et al., 2016). No efforts were made to 
investigate the long-time unsteady free-surface waves produced by two or more ships moving with different speeds. Mousaviraad et al. (2016b) analyzed the ship-ship interaction experiments both in calm water and waves. They also ran the URANS simulations, with the free-surface boundary condition considered (Mousaviraad et al., 2016a). These represent CFD's current capabilities, albeit computationally demanding. The present work explores the effects of free surface on interaction beyond the interaction forces themselves. The result of freesurface elevation was neither measured in the model tests nor presented in the CFD simulations. The demand in computational power of these CFD methods when more than one ship is in motion can be the bottleneck if real-time applications should be needed.

All the afore-mentioned studies adopted the assumption that the encountering or overtaking speed is low. Therefore, the unsteady free-surface wave effect is not essential. This assumption significantly reduces the complexity of unsteady ship-to-ship problem. However, in real maneuvering practice, the encounter speed is not always low. The importance of free-surface effects is determined by whether or not the far-field waves generated by one ship could propagate to the other ships. At lower Froude number, the amplitude of the far-field waves is very small. These waves are dissipated before they propagate to the far field, as shown in Fig. 1a. Fig. 1b shows a sketch of the flow passing the gap between two ships. The flow is "compressed" to pass through the narrow gaps between two ships with relative higher velocity. According to Bernoulli's principle, the accelerated fluid velocity could result in a decrease in pressure distribution in the gap, therefore inducing hydrodynamic interaction forces (or moments). In this low-speed case, the free-surface elevation and the hydrodynamic interaction are mainly determined by the near-field disturbance. As the speed increases, the far-field waves can be observed visibly. The far-field wave patterns generated by two pressure disturbances moving towards opposite direction are shown in Fig. 2a. The encounter process of these two disturbances is time-dependent. It can be anticipated when a disturbance is in the other's wake region, the hydrodynamic interaction will be unavoidable. In the port or inland waterways, the hydrodynamic interaction between three-dimensional vessels is also conceivably affected by the propagation of the far-field waves. The wave elevation reflects the pressure distribution on water surface. The interaction occurs when the waves produced by a ship strike the other, therefore modifying the pressure distribution over their immersed body surfaces. Thus, the hydrodynamic interaction can be apparently observed by wave interference on free surface. Benefited from satellite-imaging technology, we can observe the wave interference phenomenon by analyzing high-resolution satellite images. The encountering and overtaking process of two real ships are shown in Fig. 2b and 2c, respectively. These images show the far-field wave interference, which indicates the ship-to-ship operation is not only limited at low Froude number. Even though the transverse separation between the ships is large, the wave interference effect can still result in strong hydrodynamic interaction. A rigid free-surface assumption is not capable of predicting the hydrodynamic interactions induced by far-field waves. A new methodology should be proposed to deal with the relevant free-surface boundary condition.

The main challenge of imposing a non-rigid free-surface condition arises from the speed term in the body boundary condition (see. Eq. (16) later). For multiple ships travelling with various speeds, it is not possible to express the free-surface boundary condition by a single velocity potential (unless one uses 
an earth-fixed coordinate system as in Yeung (1975)). A superposition method,

148 however, can be applied to account for the velocity potentials induced by each 149 vessel with its own, distinctive speed. In order to account for the different speeds appearing in free-surface boundary condition, Yuan et al. (2015) proposed an uncoupled method based on the superposition principle. Therein, the speed difference of two ships was assumed to be small. Thus, the free-surface condition could be treated (arguably) as two steady-state problem, one for each ship. This method is not applicable to predict the interaction forces when ships' speeds are not the same, or when two ships are moving towards each other. In these cases, the unsteady effects become essential and the time-dependent terms must be taken into account. In the present study, we will extend Yuan's work to the time domain and discuss the importance of free-surface effects on a multi-ship problem.

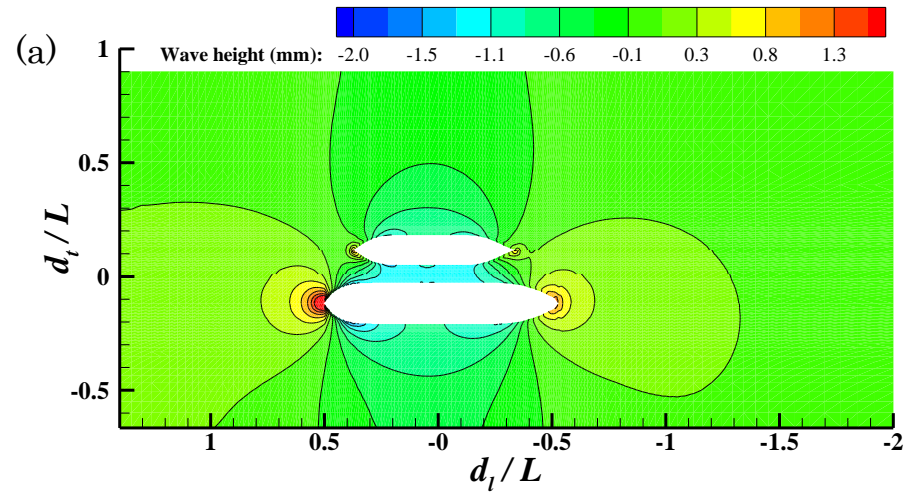

(b)

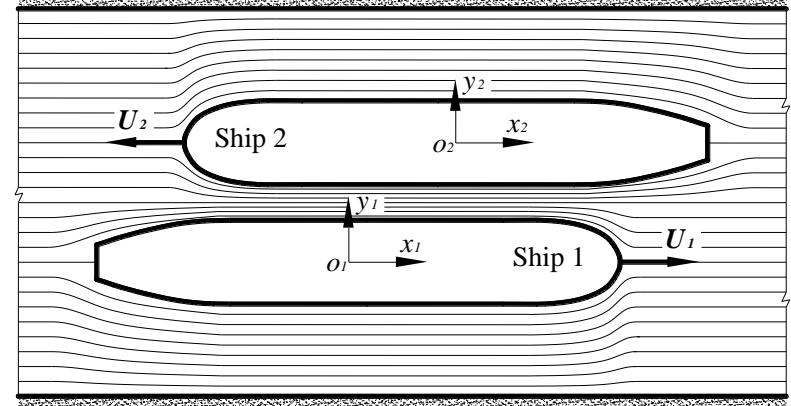

160 Fig. 1. (a), wave patterns produced by two ships travelling at low Froude number $\left(F_{n}=\right.$ 0.043) (Yuan et al., 2015). (b), sketch of flow passing the gap between two ships.

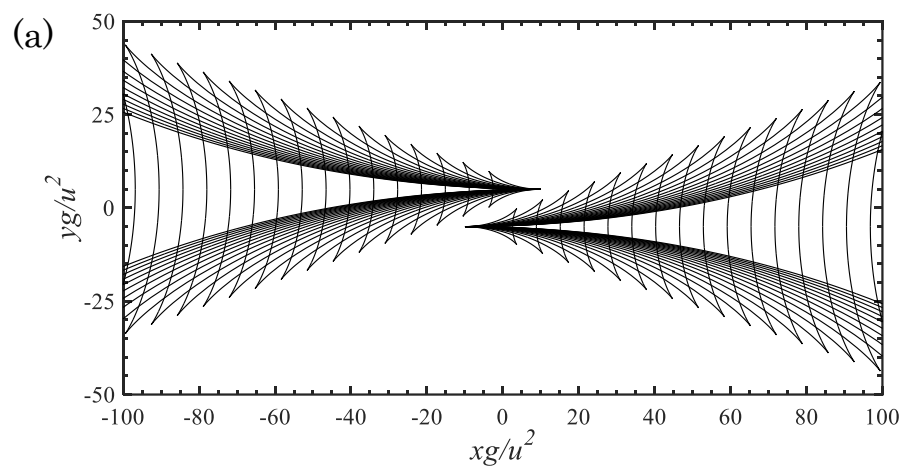


(b)

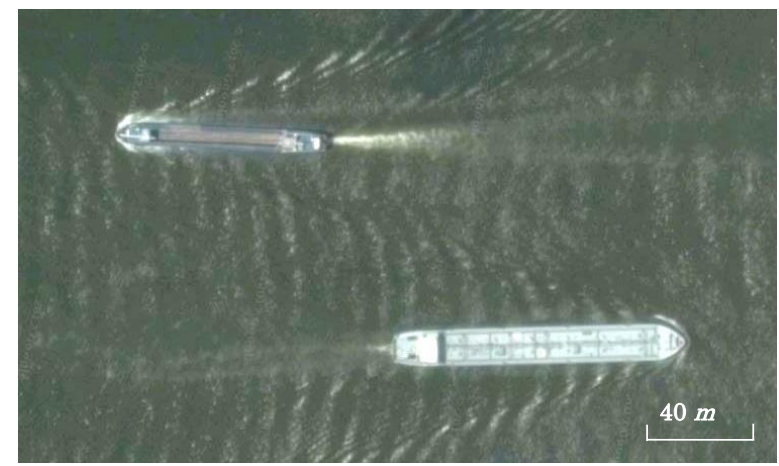

(c)

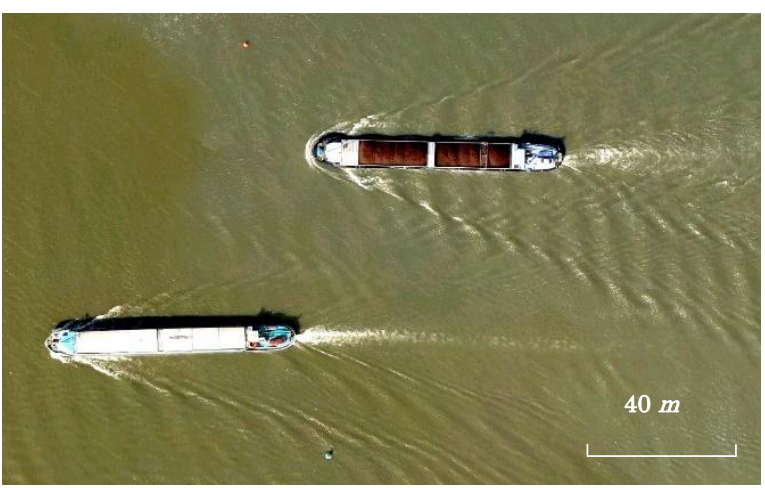

Fig. 2. (a), sketch showing the transverse and divergent waves generating by two pressure disturbances moving towards opposite direction. (b) and (c), satellite image of ship wakes taken from the Google Earth database. (b), two ships encountering at Dordtse Kil, The Netherlands (https://www.google.com/maps/@51.7519406,4.6291446,652a,35y,75.15h,8.71t/data=!3 m1!1e3?hl=en). (c), two ships overtaking at Lek, Netherlands

(https://www.google.com/maps/@51.9953387,5.0694056,298a,35y,325.35h/data=!3m1!1 $\mathrm{e} 3$ ?hl=en). The Froude number of the vessels in the lower part of (b) and upper part of (c) is $F_{n} \approx 0.15$.

\section{THE BOUNDARY-VALUE PROBLEM}

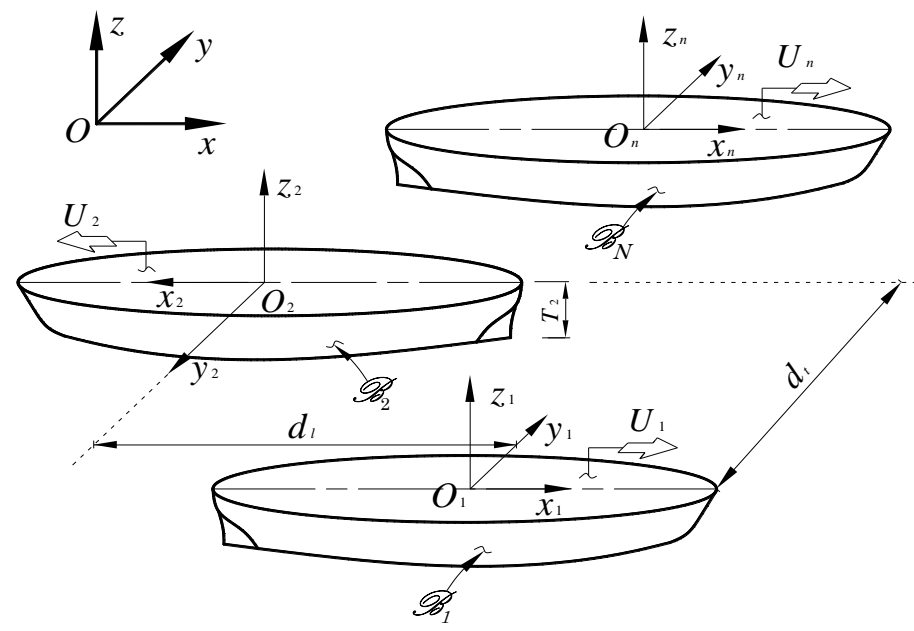

Fig. 3. Coordinate systems.

Consider $N$ ships moving at speeds $U_{j}(j=1,2, \ldots, N)$ with respect to a spacefixed reference frame $\mathbf{x}=(x, y, z)$ in an inviscid fluid of depth $h$ as shown in Fig. 3. A right-handed Cartesian coordinate system $\mathbf{x}_{\mathbf{j}}=\left(x_{j}, y_{j}, z_{j}\right)(j=1,2, \ldots, N)$ is fixed to each ship with its positive $x_{j}$-axis pointing towards the bow, positive $Z$ 
axis pointing upwards and $z_{j}=0$ being the undisturbed free-surface. Let $\Phi$ (x, $t)$ be the velocity potential describing the disturbances generated by the forward motion of the ships and $\zeta(x, y, t)$ be the free-surface wave elevation. In the fluid domain, the total velocity potential $\Phi$ satisfies the Laplace equation

$$
\nabla^{2} \Phi(\mathbf{x}, t)=0 .
$$

The fluid pressure, $p(\mathbf{x}, t)$, is given by Bernoulli's equation

$$
p(\mathbf{x}, t)=-\rho\left(\frac{\partial \Phi}{\partial t}+\frac{1}{2} \nabla \Phi \cdot \nabla \Phi+g z\right)+p_{0},
$$

where $\rho$ is the fluid density, $p_{0}$ is the atmospheric pressure, which is used as a reference pressure and assumed to be constant. Assuming there is no overturning and breaking waves on the free-surface, we can use this Eulerian description of the flow to describe the free-surface motion. The free-surface elevation is given by $z=\zeta(x, y, t)$. A fluid particle on the free-surface is assumed to stay on the free-surface, which leads to the following kinematic free-surface boundary condition:

$$
\frac{D}{D t}(\zeta-z)=0, \text { on } z=\zeta .
$$

The material derivative in Eq. (3) is given by:

$$
\frac{D}{D t}=\frac{\partial}{\partial t}+\nabla \Phi \cdot \nabla
$$

The dynamic free-surface condition is that the fluid pressure equals the constant atmospheric pressure $p_{o}$ on the free-surface, since the position of the freesurface is unknown. According to Bernoulli's equation Eq. (2), the dynamic freesurface boundary condition can be written as

$$
\frac{\partial \Phi}{\partial t}+\frac{1}{2} \nabla \Phi \cdot \nabla \Phi+g z=0, \text { on } z=\zeta .
$$

By applying Taylor series expanded about $z=0$ and only keeping the linear terms, the dynamic and kinetic free-surface conditions can be linearized as

$$
\frac{\partial \zeta}{\partial t}-\frac{\partial \Phi}{\partial z}=0, \text { on } z=0
$$

$$
\frac{\partial \Phi}{\partial t}+g \zeta=0, \text { on } z=0 .
$$

Combining Eq. (6) and (7), we obtain the free-surface boundary condition:

$$
\frac{\partial^{2} \Phi}{\partial t^{2}}+g \frac{\partial \Phi}{\partial z}=0, \text { on } z=0 .
$$

It should be noted the free-surface $\zeta$ can be found from Eq. (7) when the velocity potential $\Phi$ is known. On the wetted body surface, the no-flux boundary conditions are used, and the following 'exact' boundary condition can be formulated:

$$
\frac{\partial \Phi}{\partial n}=U_{j}\left(n_{x}\right)_{j}, \text { on } \mathscr{B}_{j}, j=1,2, \ldots, N
$$


where $\partial / \partial n$ is the derivative along the normal vector $\mathbf{n}=\left(n_{x}, n_{y}, n_{z}\right)$ into the hull surface. We choose the normal vector to be positive into the fluid domain.

Assuming the disturbance of the fluid is small, we represent the total velocity potential produced by the presence of all ships in the fluid domain in a spacefixed frame to satisfy the following superposition principle:

$$
\Phi(\mathbf{x}, t)=\sum_{j=1}^{N} \Phi_{j}(\mathbf{x}, t), j=1,2, \ldots, N
$$

where $\Phi_{j}(\mathbf{x}, t)$ is the velocity potential produced by the presence of ship $j$ moving with $U_{j}$, while the remaining ships are momentarily stationary in this frame. For the linear problem, the body-fixed coordinate system $\mathbf{x}_{\mathbf{j}}=\left(x_{j}, y_{j}, z_{j}\right)(j=1,2$, $\ldots, N)$ is used to solve the BVP for $\mathrm{N}$ vessels in concurrent motion. The relation between the body- and space-fixed coordinate system is straightforward, viz.

$$
x_{j}=x-U_{j} t, j=1,2, \ldots, N
$$

Let $\phi_{j}\left(\mathbf{x}_{\mathbf{j}}, t\right)$ represents $\Phi_{j}(\mathbf{x}, t)$ in the body-fixed coordinate system, the following relation can be obtained

$$
\frac{d \Phi_{j}}{d t}=\left(\frac{\partial}{\partial t}-U_{j} \frac{\partial}{\partial x_{j}}\right) \phi_{j}
$$

The velocity potential $\phi_{j}$ satisfies the Laplace equation and body 'exact' boundary condition:

$$
\nabla^{2} \phi_{j}\left(\mathbf{x}_{\mathbf{j}}, t\right)=0, j=1,2, \ldots, N
$$

$$
\frac{\partial \phi_{j}}{\partial n}=\delta_{i j} U_{j}\left(n_{x}\right)_{j}, \text { on } \mathscr{B}_{i}, i, j=1,2, \ldots, N
$$

The Kronecker delta $\delta_{i j}$ is the quantity defined by

$$
\delta_{i j}= \begin{cases}1 & i=j \\ 0 & i \neq j\end{cases}
$$

Substituting Eq. (12) into the linearized free-surface condition in Eq. (8), we obtain the linearized free-surface condition in the body-fixed coordinate system

$$
\frac{\partial^{2} \phi_{j}}{\partial t^{2}}-2 U_{j} \frac{\partial^{2} \phi_{j}}{\partial x_{j} \partial t}+U_{j}^{2} \frac{\partial^{2} \phi_{j}}{\partial x_{j}^{2}}+g \frac{\partial \phi_{j}}{\partial z_{j}}=0, \text { on } z=0
$$

The boundary condition on the sea bottom and side walls, if any, can be expressed as

$$
\frac{\partial \phi_{j}}{\partial n}=0
$$

Besides, a radiation condition is imposed on the control surface to ensure that waves vanish at upstream infinity

$$
\phi_{j} \rightarrow 0, \quad \zeta_{j} \rightarrow 0 \text { as } \sqrt{x_{j}^{2}+y_{j}^{2}} \rightarrow \infty
$$


where $\zeta_{j}$ is the wave elevation as seen in the $j$-th body-fixed frame and is given 241 by Eq.(30).

\section{NUMERICAL SOLUTIONS}

243 Eqs. (13) - (18) define a complete set of BVP. Each one of BVP is time-dependent 244 but can be solved individually and independently; only a single speed of ship $j$ 245 appears in the free-surface condition in Eq.(16). The coupled problem is decoupled into $N$ independent BVPs. At each time instant, the BVP in Eqs. (13) - (18) can be solved numerically. Following the work of Hess \& Smith (1964), the boundaries are discretized into a number of quadrilateral panels with constant source density $\sigma\left(\xi_{j}\right)$, where $\xi_{j}=\left(\xi_{j}, \eta_{j}, \varsigma_{j}\right)$ is a position vector on the boundaries in the $j$-th body-fixed frame and the free-surface (Bai \& Yeung, 1974). Let $\mathbf{x}_{\mathbf{j}}=$ $\left(x_{j}, y_{j}, z_{j}\right)$ denote a point inside the fluid domain or on the boundary surface, the velocity potential $\phi$ can be expressed by a source distribution on the boundary of the fluid domain

$$
\phi\left(\mathbf{x}_{\mathbf{j}}\right)=\iint_{S_{f}+S_{c}+\sum_{j=1}^{n} S_{b j}} \sigma\left(\boldsymbol{\xi}_{\mathbf{j}}\right) G\left(\mathbf{x}_{\mathbf{j}}, \boldsymbol{\xi}_{\mathbf{j}}\right) d s, \quad j=1,2, \ldots, N
$$

where $G=1 / r$ is the Rankine-type source function, with $r$ being the distance between $\xi_{j}$ and $\mathbf{x}_{\mathbf{j}} . S_{f}, S_{c}$ and $S_{b}$ indicate the free-, control- and body-surface respectively. More detailed numerical implementation on the solution of BVP can be found in Yuan et al. (2014b). The same in-house developed program "MHydro" is deployed in the present study as the framework to investigate ship hydrodynamics in restricted waterways. Special care should be taken to implement a suitable open boundary condition to satisfy Eq. (18). In numerical calculations, the computational domain is always truncated at a distance away from the ship hull. In general, waves will be reflected from the truncated boundaries and contaminate the flow in the computational domain. In the present study, a secondorder upwind difference scheme is applied on the free-surface to obtain the time and spatial derivatives:

$$
\frac{\partial^{2} \phi_{j}}{\partial x^{2}}\left(\left(\mathbf{x}_{\mathbf{j}}\right)_{k}\right)=\frac{1}{\Delta x^{2}}\left(\frac{1}{4} \phi_{j}\left(\left(\mathbf{x}_{\mathbf{j}}\right)_{k+4}\right)-2 \phi_{j}\left(\left(\mathbf{x}_{\mathbf{j}}\right)_{k+3}\right)+\frac{11}{2} \phi_{j}\left(\left(\mathbf{x}_{\mathbf{j}}\right)_{k+2}\right)-6 \phi_{j}\left(\left(\mathbf{x}_{\mathbf{j}}\right)_{k+1}\right)+\frac{9}{4} \phi_{j}\left(\left(\mathbf{x}_{\mathbf{j}}\right)_{k}\right)\right)
$$

Here $k$ refers to the index for the panels. According to Bunnik (1999) and Kim et al. (2005), and earlier works, Eq. (18) can be satisfied effectively by applying Eq. (20). It should be noted that such a $2^{\text {nd-order } u p w i n d ~ d i f f e r e n c i n g ~ s c h e m e ~ i s ~}$ applied at each body-fixed frame locally. This is essential to deal with ships moving in opposite directions.

For each individual velocity potential $\phi_{j}$, the BVP is unsteady due to the timedependent terms in Eq. (16). In previous studies on ship-to-ship interaction problems (Yeung, 1978; Yeung and Tan, 1980, Xu et al., 2016), within the framework of potential-flow theory, the BVP was not posed in the time domain as the free-surface was assumed to be rigid. It was solved independently at each individual time step. The unsteady effects need only be considered in the pressure calculations in Eq. (27). The unsteady interaction forces calculated in these studies are not exactly 'unsteady', since the velocity potential at each time step is not time dependent. The velocity potential obtained at $t_{n}$ is not related to that obtained at $t_{n-1}$, and it will also not determine that at $t_{n+1}$. In the present study, 
the unsteady BVP will be solved in the time domain by an iteration scheme. The essential steps are:

1. Determine the initial condition. We assume that at the initial stage of ship-to-ship operation, the moving ships are sufficiently far apart so that their interactions are initially negligible. Thus, the time-dependent terms are removed from the free-surface condition in Eq. (16), and we have

$$
U_{j}^{2} \frac{\partial^{2}\left(\phi_{j}^{k}\right)^{*}}{\partial x_{j}^{2}}+g \frac{\partial\left(\phi_{j}^{k}\right)^{*}}{\partial z_{j}}=0
$$

Here $\left(\phi_{j}^{k}\right)^{*}$ is the time-independent velocity potential at the time step $k$. The computational domain and the corresponding panel distribution at each time step $k$ can be constructed and the steady BVP in Eqs. (13) to (15), (21), (17) and (18) can be solved straightforwardly by using the Rankine-source panel method. The time-independent velocity potential $\left(\phi_{j}^{k}\right)^{*}$ can be obtained, which will be used as the initial guess to calculate the time derivatives of unsteady velocity potential $\phi_{j}^{k}$ in Eq. (22).

2. By applying the second-order backward difference scheme, the time derivatives in Eq. (16) can be calculated according to the following formulas

$$
\frac{\partial \phi_{j}^{k}}{\partial t}=\frac{1}{\Delta t}\left(\frac{3}{2}\left(\phi_{j}^{k}\right)^{*}-2\left(\phi_{j}^{k-1}\right)^{*}+\frac{1}{2}\left(\phi_{j}^{k-2}\right)^{*}\right)
$$

$$
\frac{\partial^{2} \phi_{j}^{k}}{\partial t^{2}}=\frac{1}{\Delta t^{2}}\left(2\left(\phi_{j}^{k}\right)^{*}-5\left(\phi_{j}^{k-1}\right)^{*}+4\left(\phi_{j}^{k-2}\right)^{*}-\phi_{j}^{*}\left(\phi_{j}^{k-3}\right)^{*}\right)
$$

3. By, substituting Eq. (22) into Eq. (16), the following time-domain freesurface condition can be obtained

$$
\frac{\partial^{2} \phi_{j}^{k}}{\partial t^{2}}-2 U_{j} \frac{\partial \phi_{j}^{k}}{\partial t} \cdot \frac{\partial \phi_{j}^{k}}{\partial x_{j}}+U_{j}^{2} \frac{\partial^{2} \phi_{j}^{k}}{\partial x_{j}^{2}}+g \frac{\partial \phi_{j}^{k}}{\partial z_{j}}=0
$$

Solving the unsteady BVP in Eqs. (13) to (15), (23), (17) and (18), we can obtain the unsteady velocity potential $\phi_{j}^{k}$. Residual errors of time derivatives of $\left|\left(\phi_{j}^{k}\right)^{*}-\phi_{j}^{k}\right|$ can be evaluated. If $\left|\left(\phi_{j}^{k}\right)^{*}-\phi_{j}^{k}\right|<\varepsilon$, the iteration stops and $\phi_{j}^{k}$ will be used to calculate the pressure and wave elevation. Otherwise, $\left(\phi_{j}^{k}\right)^{*}$ in Eq. (22) will be replaced by the newly obtained $\phi_{j}^{k}$, and the iteration continues until $\left|\left(\phi_{j}^{k}\right)^{*}-\phi_{j}^{k}\right|<\varepsilon$. It is known that the iterative scheme has advantages of high accuracy and good numerical stability.

Once the unknown potential $\phi_{j}$ is solved on the plane $z=0$ and on the body $\mathcal{B}_{j}$, the unsteady pressure components under its individual coordinate system can be obtained from linearized Bernoulli's equation

$$
\left.p_{j}\right|_{\mathbf{x}_{\mathrm{j}}}=-\rho\left[\left.\frac{\partial \phi_{j}}{\partial t}\right|_{\mathbf{x}_{\mathrm{j}}}-\left.U_{j} \frac{\partial \phi_{j}}{\partial x_{j}}\right|_{\mathbf{x}_{\mathbf{j}}}\right], j=1,2, \ldots, N
$$


We should point out that because of the first unsteady term in Eq. (24), the total pressure $p$ in coordinate system $\mathbf{x}_{\mathbf{j}}$ cannot be expressed directly as the sum of all the pressure components in each of their local frames. To transfer the pressure from coordinate system $\mathbf{x}_{\mathbf{i}}$ to $\mathbf{x}_{\mathbf{j}}$, the following relation needs to be observed

$$
\left.\frac{d \phi_{i}}{d t}\right|_{\mathbf{x}_{\mathrm{j}}}=\left.\left(\frac{\partial}{\partial t}-\left(U_{j}-U_{i}\right) \frac{\partial}{\partial x_{i}}\right) \phi_{i}\right|_{\mathbf{x}_{\mathrm{j}}}, i, j=1,2, \ldots, N
$$

It should be noted that the partial derivative symbol of the first term in Eq. (24) is retained to make it consistent with Eq. (12) where the potential is expressed in the body-fixed coordinate system $\mathbf{x}_{\mathbf{j}}$. Note however, the body-fixed coordinate system $\mathbf{x}_{\mathbf{j}}$ turns out to be in the reference frame for the other body-fixed coordinate system $\mathbf{x}_{\mathbf{i}}$. Therefore, $\frac{\partial \emptyset_{j}}{\partial t}$ is actually calculated as a total derivative by using Eq. (25). The unsteady pressure in coordinate system $\mathbf{x}_{\mathbf{i}}(i=1,2, \ldots, N, i \neq j$ ) can then be 'transferred' to $\mathbf{x}_{\mathbf{j}}$ as

$$
\left.p_{i}\right|_{\mathbf{x}_{\mathrm{j}}}=-\rho\left[\left.\left(\frac{\partial}{\partial t}-\left(U_{j}-U_{i}\right) \frac{\partial}{\partial x_{i}}\right) \phi_{i}\right|_{\mathbf{x}_{\mathrm{j}}}-\left.U_{i} \frac{\partial \phi_{i}}{\partial x_{i}}\right|_{\mathbf{x}_{\mathrm{j}}}\right]=-\left.\rho\left(\frac{\partial}{\partial t}-U_{j} \frac{\partial}{\partial x_{i}}\right) \phi_{i}\right|_{\mathbf{x}_{\mathrm{j}}}, i, j=1,2, \ldots, N
$$

Note the subtle differences in the subscripts between Eq. (24) and (26). The total pressure $p$ in coordinate system $\mathbf{x}_{\mathbf{j}}$ can be written as

$$
\left.p\right|_{\mathbf{x}_{\mathrm{j}}}=\left.\sum_{i=1}^{N} p_{i}\right|_{\mathbf{x}_{\mathrm{j}}}=-\left.\rho \sum_{i=1}^{N}\left(\frac{\partial}{\partial t}-U_{j} \frac{\partial}{\partial x_{i}}\right) \phi_{i}\right|_{\mathbf{x}_{\mathrm{j}}}, i, j=1,2, \ldots, N
$$

Integrating the pressure over the hull surface, we can express the forces (or moments) on the $i$ th hull induced by the $j$ th ship as:

$$
F_{k}^{j}=\iint_{B_{j}} p n_{k} d S, j=1,2, \ldots, N
$$

where $k=1,2, \ldots, 6$, representing the force in surge, sway, heave, roll, pitch and yaw directions, and

$$
n_{k}= \begin{cases}\mathbf{n}, & k=1,2,3 \\ \mathbf{x} \times \mathbf{n}, & k=4,5,6\end{cases}
$$

The free-surface elevation can be obtained from dynamic free-surface boundary condition in Eq. (7). Similar to the pressure expression, the unsteady wave elevation in coordinate system $\mathbf{x}_{\mathbf{i}}(i=1,2, \ldots, N, i \neq j)$ can be transferred to $\mathbf{x}_{\mathbf{j}}$ as

$$
\left.\zeta_{i}\right|_{\mathbf{x}_{\mathrm{j}}}=-\left.\frac{1}{g}\left(\frac{\partial}{\partial t}-U_{j} \frac{\partial}{\partial x_{i}}\right) \phi_{i}\right|_{\mathbf{x}_{\mathrm{j}}}, i, j=1,2, \ldots, N
$$

The total wave elevation in coordinate system $\mathbf{x}_{\mathbf{j}}$ can be written as

$$
\left.\zeta\right|_{\mathbf{x}_{\mathrm{j}}}=-\left.\frac{1}{g} \sum_{i=1}^{N}\left(\frac{\partial}{\partial t}-U_{j} \frac{\partial}{\partial x_{i}}\right) \phi_{i}\right|_{\mathbf{x}_{\mathrm{j}}}, i, j=1,2, \ldots, N
$$

We note that we have not imposed a Kutta condition at the stern, as a first approximation, or equivalently, the stern is considered pointed.

\section{VALIDATIONS OF NUMERICAL MODEL}


The convergence study is carried out on two identical Wigley III hulls in headon encounter. We calculate the lateral force and wave elevation to exam the convergence of the superposition method with different time steps $(\Delta t)$. The panel size to ship length ratio at each Froude number is fixed at $\Delta x / L=1 / \kappa$. The time then can be non-dimensionalized by

$$
t^{\prime}=\Delta x / U=\frac{1}{\kappa F_{n}} \sqrt{\frac{L}{g}}
$$

In the present study, $\kappa=60$ was found adequate to obtain a convergent result. The results shown in Fig. 4 confirm the convergence of the present superposition method by reducing the time stepping. It should be noted that the convergence becomes slower as the encounter speed increases.

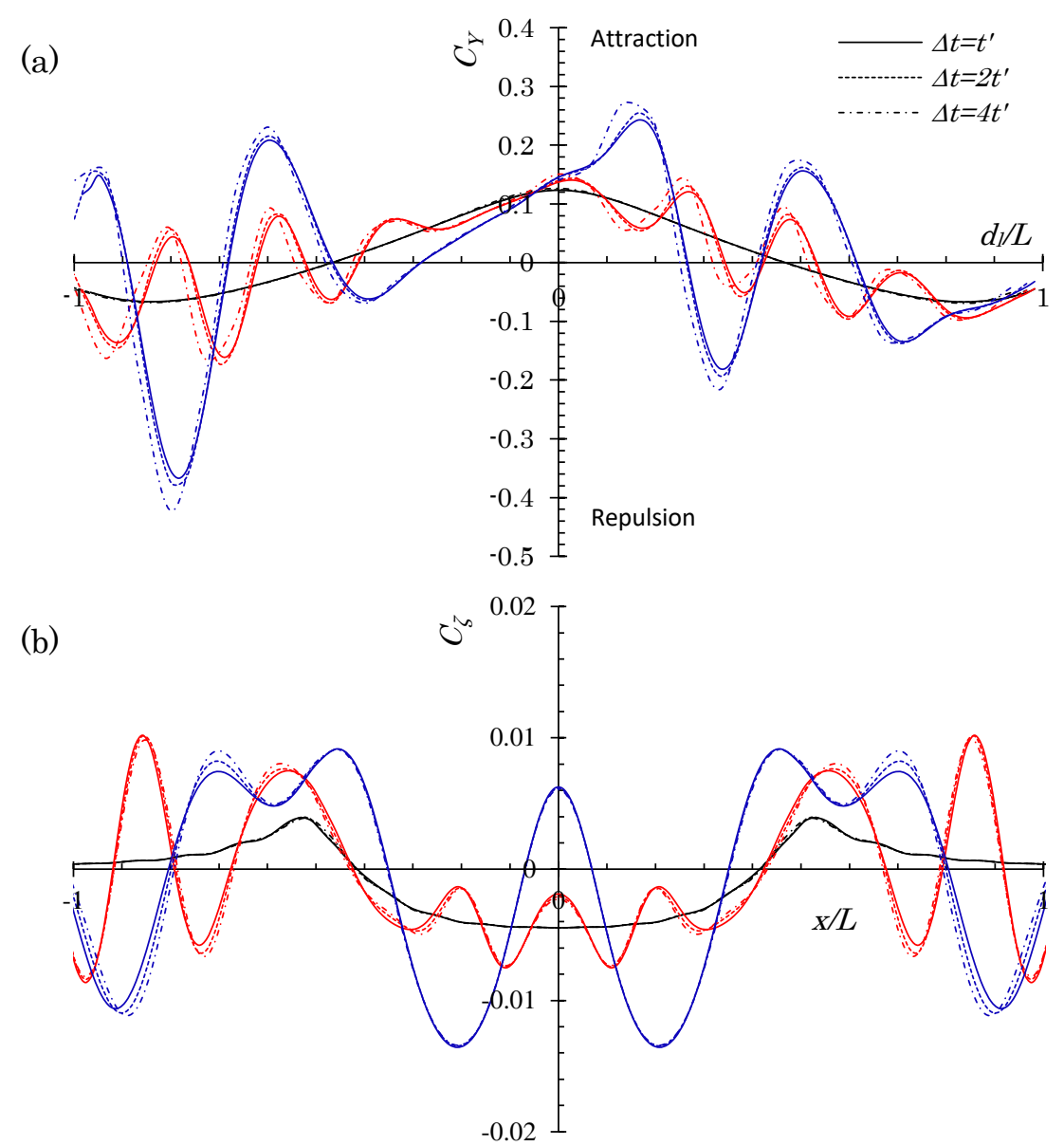

Fig. 4. Convergence study on two identical Wigley III hulls (Journee, 1992) in head-on encounter with $d_{t} / B=2, d_{t}$ being the lateral separation between two ships (a) Sway force; (b) wave profile at the center line between two ships at the moment of side-by-side configuration $\left(d_{F}=0\right)$. The black, red and blue cures correspond to $F_{n}=0.1,0.2$ and 0.3 respec- $^{-}$ tively. $C_{Y}$ and $C_{\zeta}$ is non-dimensionalized by $\frac{1}{2} \rho B T\left|U_{1} U_{2}\right|$ and by $2 \pi\left|U_{1} U_{2}\right| / g$ respectively.

Model-test data on ship-to-ship interaction with different speeds as a parameter is rather rare. To run the tests, an auxiliary carriage must be installed, in addition to the main tow carriage. Therefore, the encountering tests were not included in Oltmann (1970). In the present study, as another check, the benchmark data published by Vantorre, et al. (2002) is used to validate the numerical results of the encountering cases. Two ship models with scale factor $1 / 75$ are 
used for encountering or overtaking tests (referred as Model D and Model E). The main particulars of Model D $(j=2)$ and Model E $(j=1)$ in model scale can be found in Table 1. In the model test, Model $\mathrm{E}$ was towed by the main carriage along the center line $(y=0)$ of the tank, while Model D was towed by an auxiliary carriage. The transverse separation is $d t=B_{D}+0.5 B_{E}$ and the water depth $d$ is $0.248 m$.

Table 1. Main particulars of Model D and Model E in Vantorre, et al. (2002).

\begin{tabular}{lll}
\hline & Model E $(j=1)$ & Model D $(j=2)$ \\
\hline Length $(m)$ & $L_{E}=3.824$ & $L_{D}=3.864$ \\
Breadth (m) & $B_{E}=0.624$ & $B_{D}=0.55$ \\
Draft $(m)$ & $T_{E}=0.207$ & $T_{D}=0.18$ \\
Block coefficient & $C_{B E}=0.816$ & $C_{B D}=0.588$
\end{tabular}

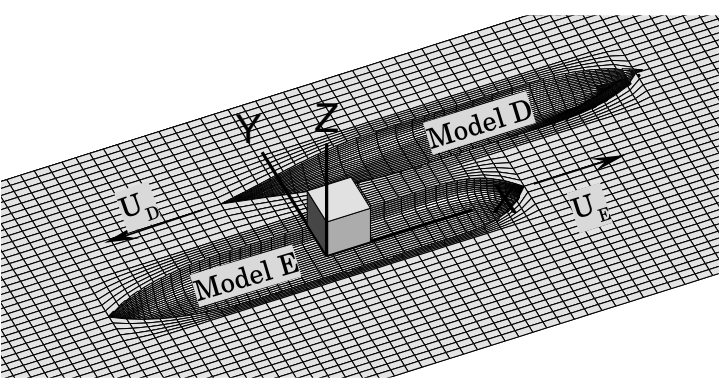

Fig. 5. Panel distribution on partial computational domain. There are 9,950 panels distributed on the entire computational domain: 1,900 on the wetted body surface of Model E, and 2,170 on the wetted body surface of Model D, 5,880 on the free-surface. The free-surface is truncated at $2 L_{E}$ upstream and $2 L_{E}$ downstream with regard to body-fixed frame on Model E.

Fig. 5 is the mesh distribution on the partial computational domain when Model E encounters Model D. It should be noted that the side walls of the tank are not modeled. In order to minimize the panel number, the free surface is truncated at $0.27 L_{E}$ and $0.42 L_{E}$ laterally with regard to Model D and Model E respectively. In calm water test, it has been proved by Yuan and Incecik (2016b) that the side wall effects are negligible at $d_{s b} / L>0.25$ and $F_{n}<0.25$. It should also be noted that in the encountering simulation, the longitudinal separation $d_{l}$ is measured in body-fixed frame on Model E. The longitudinal separation between two ships at the moment shown in Fig. 5 has a positive sign. The time step $\Delta t$ in the numerical calculation is $0.18 \mathrm{~s}$. The numerical results, as well as the experimental measurements, are shown in Fig. 6-Fig. 9.

Fig. 6 shows the interactions forces on Model $\mathrm{E}$ at $F_{n}=0$ passed by Model D at $F_{n}=0.078$. In engineering practice, this case study aims to investigate the mooring forces induced by a passing ship in the harbor areas or inland waterways. Fig. 7 and Fig. 8 shows the interaction forces on Model $\mathrm{E}$ at $F_{n}=0.039$ and $F_{n}=0.078$ encountered by Model D at $F_{n}=0.078$. These two case studies aim to validate the feasibility of the present superposition method on simulating the ships moving towards opposite directions. Fig. 9 shows the interactions forces on Model E at $F_{n}=0.078$ overtaken by Model D at $F_{n}=0.117$. In all of these four cases, the forces on both ships are calculated numerically by the described methodology. However, only the forces on Model E, which was towed by the main carriage, were measured in model tests. Generally, the agreement between this 
version of potential-flow solver (MHydro) and experimental measurement is very good. It indicates that the potential-flow method is applicable to predict the hydrodynamic interactions between two ships with different forward speeds.

It is found from subfigures (a) of Fig. 6 to Fig. 9 that the axial or longitudinal force $\left(F_{1}\right)$ is overestimated by the present potential-flow solver, even though the viscous effect is not taken into account. It indicates the hydrodynamic interaction force plays a dominate role in total axial force, and the frictional component due to the viscosity is negligible in this dynamic situation. The negative values shown in these subfigures of Fig. 6 to Fig. 9 represent the forces that are opposite to the moving direction, while the positive values represents an effective thrust which is the same as the moving direction. An interesting finding is that a very large thrust force is observed at $d_{l} / L_{E}=-0.5$ during the passing and encountering maneuvering. Physically it can be explained that before passing and encountering $\left(0<d_{l} / L_{E}<1\right)$, the presence of the other moving vessel slows the water from spreading evenly into the surrounding field. As a result, the pressure distributed over ship bow increases. At the same time, the pressure distributed over ship stern retains the same level. An increased axial force or "resistance" is expected from pressure integration. After encountering $\left(-1<d_{l} /\right.$ $\left.L_{E}<0\right)$, the high pressure area transfers to the ship stern, which will correspondingly lead to an effective thrust. However, in overtaking maneuvering as shown in Fig. 9a, the thrust force is observed at $d_{l} / L_{E}=0.5$, where the bow of Model D approaches the midship of Model E longitudinally. It can be explained that before overtaking $\left(-1<d_{l} / L_{E}<0\right)$, the presence of faster ship (Model D) accelerates the fluid velocity around the stern area of Model E. As a result, the pressure distributed over ship stern decreases. At the same time, the pressure distributed over the ship bow retains the same level. An increased "resistance" is expected from pressure integral over the hull surface of Model E. After overtaking $\left(0<d_{l} / L_{E}<1\right)$, the high pressure area transfers to the ship bow, which will correspondingly lead to a propulsion force.

During the passing, encountering and overtaking process, the symmetry of the flow in the starboard and port side is violated, as expected, by the presence of the other vessel. The maximum asymmetric flow is observed when the midships of the two ships are aligned $\left(d_{l} / L_{E} \approx 0\right.$, as shown in Fig. 1$)$, and the suction force reaches its peak value (see subfigures (b) of Fig. 6 to Fig. 9). The pressure distribution is not only asymmetric along port and starboard sides, but also in bow and stern. Consequently, a yaw moment will be induced, as shown in subfigures (c) of Fig. 6 to Fig. 9. Generally, there are four peaks of yaw moment during passing and encountering maneuvering, which appear at $d_{l} / L_{E} \approx-0.6, d_{l} / L_{E} \approx$ $-0.1, d_{l} / L_{E} \approx 0.4$ and $d_{l} / L_{E} \approx 0.9$. However, in overtaking process, only three peaks are observed at $d_{l} / L_{E} \approx-0.8, d_{l} / L_{E} \approx-0.1$ and $d_{l} / L_{E} \approx 0.5$. Based on these peaks, some empirical formulas were established to model the interaction moment (Lataire et al., 2012; Vantorre et al., 2002; Varyani et al., 2002). However, as the numbers of the peaks are not predictable, the applicability of those empirical formulas can be limited. It should be noted that in ship-bank and shiplock problem, potential flow method fails to predict the sign of the yaw moment because there is lifting force caused by the cross-flow in the stern (Yuan and Incecik, 2016a). However, in ship-to-ship problem, the hydrodynamic interaction is much more important than cross-flow effects. The predictions of yaw moment by a potential flow solver are therefore reliable. 
It is also found from Fig. 6 to Fig. 9 that the interaction forces on the ship with the lower speed are larger than those on the higher speed ship. In passing operation (see Fig. 6), the hydrodynamic interaction on Model E is significant, even though Model E is stationary without forward speed. On the contrary, the interaction is relatively unimportant on the moving ship (Model D) during passing operation. It indicates that the slower ship is more likely to lose its maneuverability during passing, encountering and overtaking process.

(a)

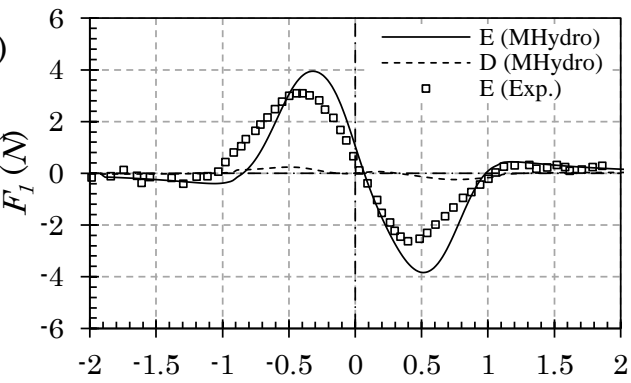

(b)

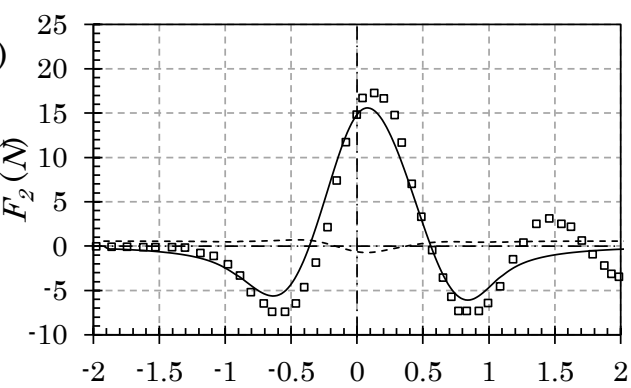

(c)

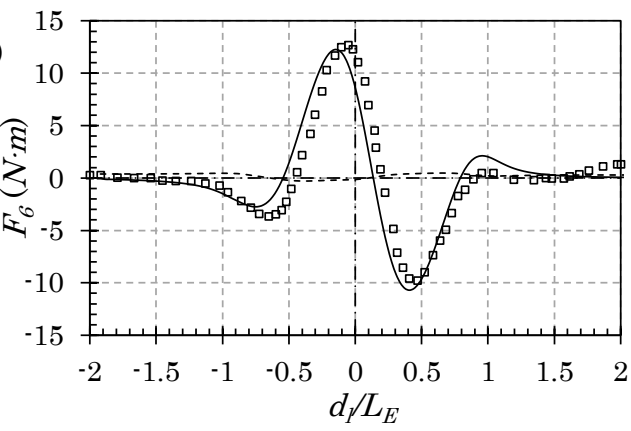

Fig. 6. (a) The axial force, (b) the sway force and (c) the yaw moment on Model E $(j=1)$ at $F_{n}=0$ passed by Model D $(j=2)$ at $F_{n}=0.078$. The positive $d_{l}$ values denote that Model D is in the upstream side of Model E. As Model D moves to the downstream side, $d_{l}$ becomes negative. EFD results are published by Vantorre et al. (2002).
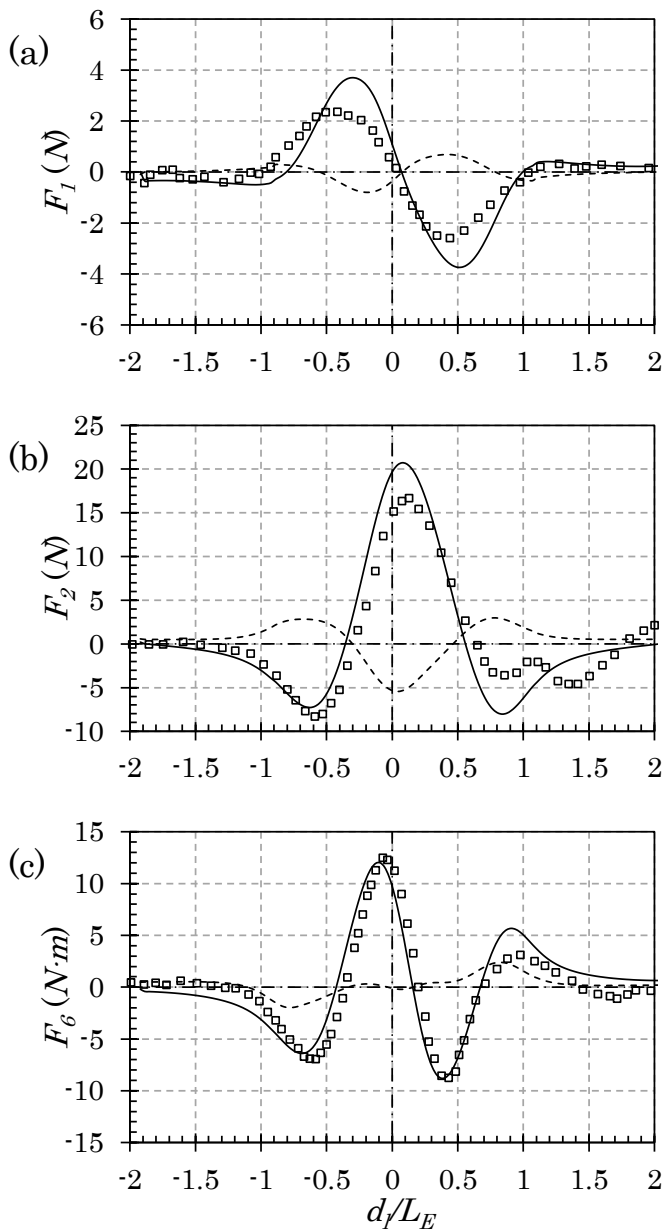

Fig. 7. (a) The axial force, (b) the sway force and (c) the yaw moment on Model E $(j=1)$ at $F_{n}=0.039$ encountered by Model D $(j=2)$ at $F_{n}=0.078$. The positive $d_{l}$ values denote that Model D is in the upstream side of Model E. As Model D moves to the downstream side, $d_{l}$ becomes negative. EFD results are published by Vantorre et al. (2002). 
(a)

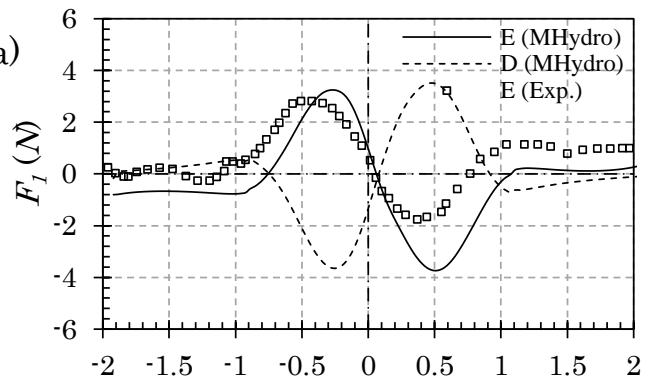

(b)

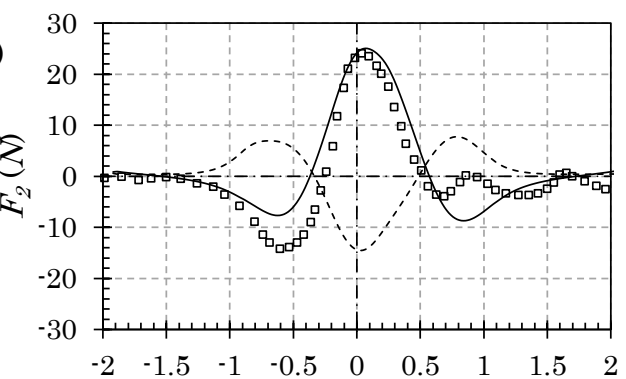

(c)

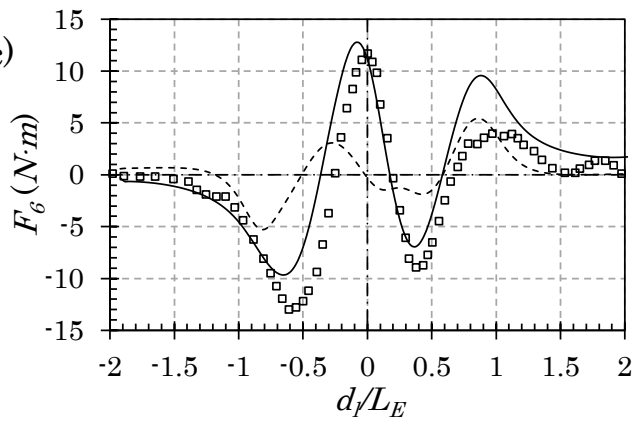

Fig. 8. (a) The axial force, (b) the sway force and (c) the yaw moment on Model E $(j=1)$ at $F_{n}=0.078$ encountered by Model D $(j=2)$ at $F_{n}=0.078$. The positive $d_{l}$ values denote that Model D is in the upstream side of Model E. As Model D moves to the downstream side, $d_{l}$ becomes negative. EFD results are published by Vantorre et al. (2002).
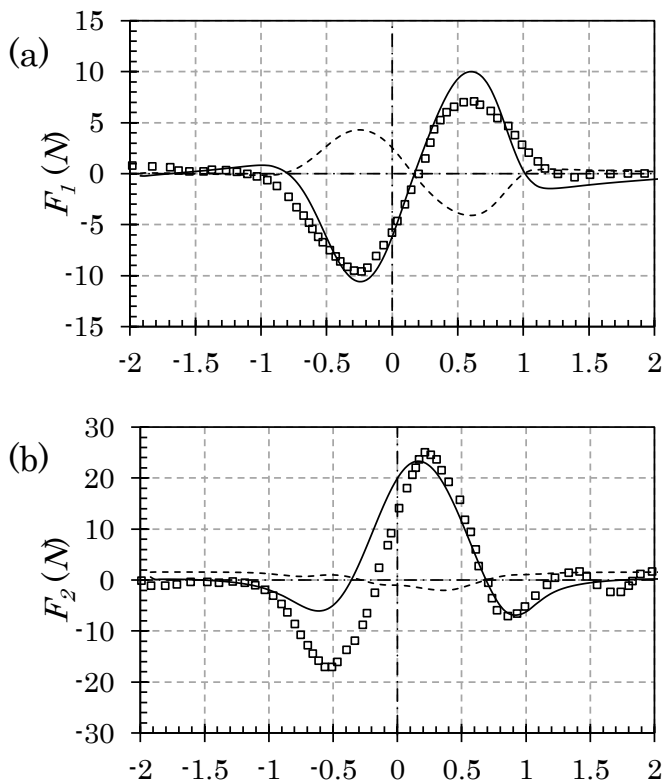

(c)

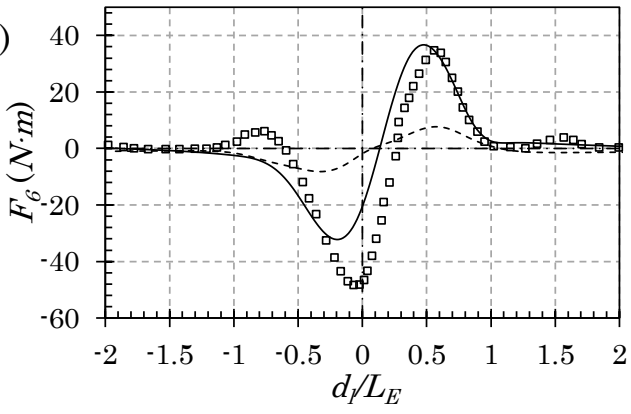

Fig. 9. (a) The axial force, (b) the sway force and (c) the yaw moment on Model $\mathrm{E}$ $(j=1)$ at $F_{n}=0.078$ overtaken by Model D $(j=2)$ at $F_{n}=0.117$. The negative $d_{l}$ values denote that Model D is in the downstream side of Model E. As Model D moves to the upstream side, $d_{l}$ becomes positive. EFD results are published by Vantorre et al. (2002).

\section{DISCUSSIONS ON FREE-SURFACE EFFECTS}

After the aforementioned validations against physical model tests, it is deemed that the predictions of the lateral force and yaw moment by a potential-flow solver are reliable. The present superposition method was extended to investigate the free-surface effects. Here, we study the interactions between two identical Wigley III hulls in head-on encounter. The geometry of the hull can be found in Journee (1992). Fig. 10 illustrates the panels distributed on the partial computational domain. The panel number per ship length $\kappa=60 . \Delta t=2 t^{\prime}$ 'is applied to all of the numerical simulations reported below. We computed the interaction forces in 6 DoF (6 Degrees of Freedom), as well as the total wave elevation. 


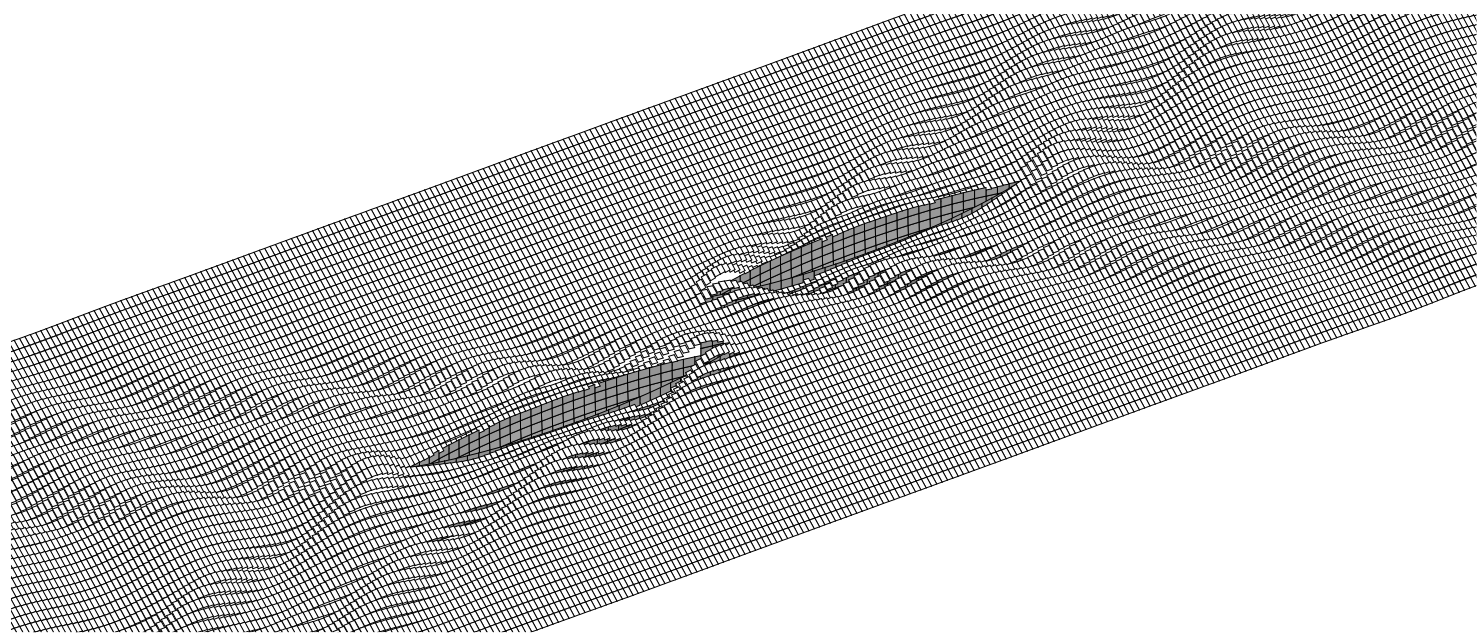

Fig. 10. Panel distribution on the computational domain of two identical Wigley III hulls in head-on encounter with $F_{n}=0.3, d_{t} / B=2$, and $d / L=1$. There are 17,760 panels distributed in the entire computational domain: 600 on the wetted body surface of each hull and 16,560 on the free surface. The computational domain is truncated at $2 L$ upstream, $2 L$ downstream and $0.5 L$ laterally with respect to the body-fixed reference frame.

\subsection{The effect of near-field disturbance and far-field waves}

Fig. 11 shows the computed lateral (sway) forces on two identical Wigley III hulls in head-on encounter with $d_{t} / B=2$. Here we compare the results obtained by using three different approaches. In the first approach, the encountering problem is treated as a steady-state problem with the steady linearized freesurface condition Eq. (21) applied but the hull boundary conditions are treated as described. Mathematically, in the pressure calculation, the first term in Eq. (27) is neglected. It is an efficient approach to deal with the steady problems, e.g., interactions between two ships travelling with the same speed (Yuan et al., 2015), or between the hulls of a catamaran or trimaran (Shahjada Tarafder and Suzuki, 2007). In the second approach, the encountering problem is treated as an unsteady problem, while a rigid-wall condition is applied on the free-surface. Mathematically, the free-surface condition in Eq. (16) is replaced by an impermeable boundary condition. The BVP therefore is solved as a problem that depends on the instantaneous configuration but no memory effects from the free surface. There are unsteady effects are coming from the time-dependent term in Eq. (27), which is related to the configuration change. Nearly all the published studies on ship-to-ship problem are based on this partially unsteady method (Korsmeyer et al., 1993; Xu et al., 2016; Yeung, 1978; Zhou et al., 2012). The advantage of this rigid-free-surface method is obvious. As the image method can be applied on the free-surface, it doesn't require panels to be distributed on the free surface. However, this method is only applicable when the speed of the ships is low. The third approach, which is method described in the present study, takes all the unsteady effects into account. The time derivatives in both Eq. (16) and Eq. (27) are considered with associated details explained. The advantage of this fully unsteady method is that it can predict the hydrodynamic interaction induced by the ship-generated waves. However, the panels need to be distributed on the free-surface, which not only increase the total mesh number, but also add difficulties to mesh up the computational domain at each time step. This latter issue is overcome by using a dynamic meshing technique at each time step. With regard to the computational time, the full method takes longer than the other two methods. As this is done within the framework of potential-flow theory, the computational time is still very manageable. Most of 
the computational efforts are spent on generating the so-called coefficient matrix (Hess and Smith, 1964) Even though it involves time iteration, the coefficient matrix retains unchanged. The time to solve the unsteady BVP for each time step is just a few minutes.

The results shown in Fig. 11 clearly demonstrate the effects of unsteady pressure and unsteady free surface. Here, we note that the unsteady pressure term in Eq. (27) is very important at all the range of encountering speeds, while the free-surface effect is only important when the encounter speed is moderate or high. Ignoring the unsteady pressure term in Eq. (27) will lead to mis-estimation of the interaction force. At $F_{n}=0.1$, the free-surface elevation and hydrodynamic interaction are mainly determined by the near-field (non-wave-like) disturbances. The rigid free-surface condition (RFC) is adequate to predict the interaction forces, as shown in Fig. 11a. As the Froude number $F_{n}$ increases to 0.2 , the far-field waves become evident, and the interaction force oscillates correspondingly, as shown in Fig. $11 \mathrm{~b}$. However, even at $F_{n}=0.2$, the interaction is still dominated by the near-field disturbance. The contribution of the force induced by far-field waves is smaller than that induced by the near-field disturbance. The fluctuations caused by the far-field waves will not deviate significantly from the near-field induced forces. The interaction force predicted by rigid free-surface condition is symmetric with respect to $d / / L=0$. But this symmetry property disappears in the presence of the far-field waves. As the far-field waves could not propagate ahead of the ship, the free-surface effect cannot be observed before the encountering taken place $\left(d_{l} / L>1\right)$. As the encountering ships are maneuvering to each other's wake region, more free-surface effect then can be observed, and some fluctuations can be observed at $d / L<1$ correspondingly. These fluctuations will not disappear (though their the amplitude will decrease) after the encountering operation. The relationship between the nearand far-field induced force is very similar to that between low- and wave-frequency surge or sway motions of a floating structure in irregular waves (Yuan et al., 2014a). The free-surface effect becomes even more significant at $F_{n}=0.3$. The force amplitude induced by the far-field waves is larger than that induced by the near-field disturbance, as can be seen in Fig. 11(c). There are only three peaks induced by near-field disturbance. However, the peaks altered by the farfield waves are not easily predictable. Therefore, the empirical formulas based on low speed model (Lataire et al., 2012; Vantorre et al., 2002; Varyani et al., 2002) cannot be considered as effective in the interaction forces when the freesurface effect becomes important. It can be concluded that the free-surface effects must be taken into account at $F_{n}>0.2$.

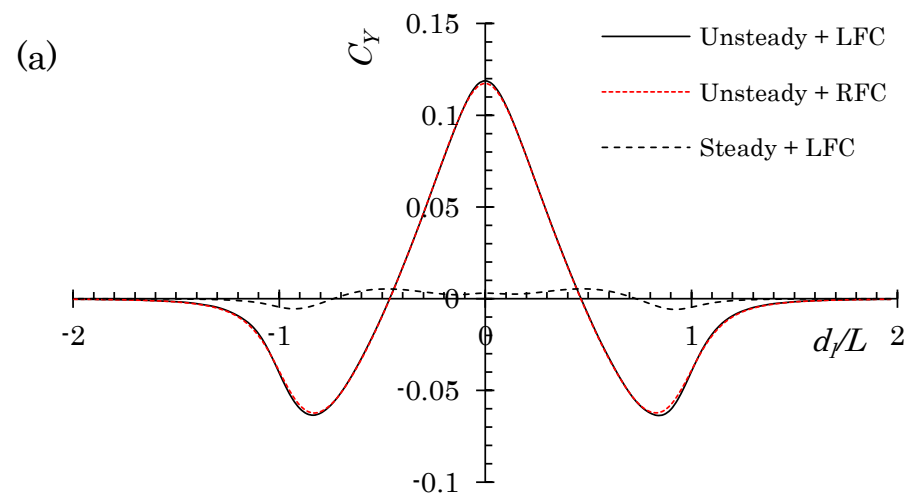



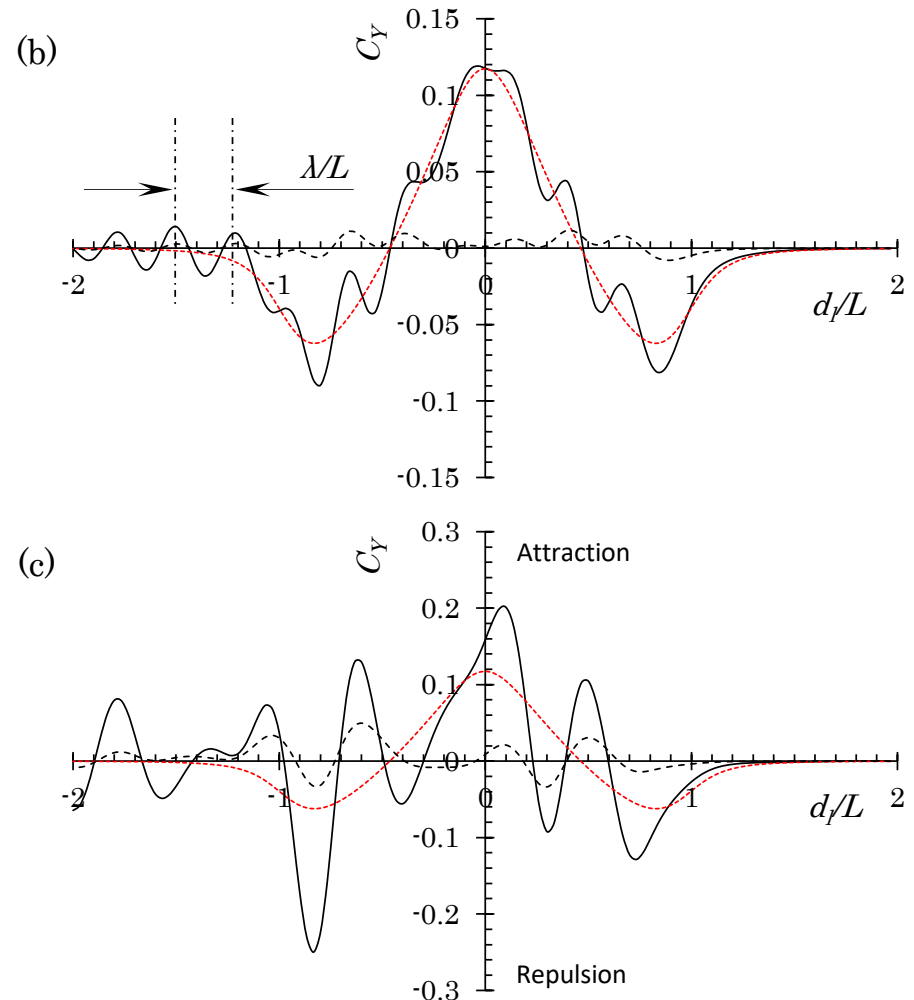

553 Fig. 11. Sway force acting on two identical Wigley III hulls in head-on encounter with $d_{t} / B=2$. (a) $F_{n}=0.1$; (b) $F_{n}=0.2$; (c) $F_{n}=0.3$. $d_{l} / L=0$ corresponds to the moment $t=t_{s}$, when the midships of the two ships are aligned. $d_{l} / L>0$ corresponds to $t<t_{s}, d_{l} / L<0$ corresponds to $t>t_{S .} C_{Y}$ is non-dimensionalized by $\frac{1}{2} \rho B T\left|U_{1} U_{2}\right|$. LFC indicates that the linearized freesurface condition is used; RFC indicates that the rigid-wall free-surface condition is used.

Fig. 12 shows the wave profile at the moment when the midships of two Wigley hulls are aligned. The labeling of 'Steady' indicates the first two terms in Eq. (16) are ignored, while 'Unsteady' indicates the BVP is solved fully in the time domain by using an iteration scheme in tine. At low Froude number $F_{n}=0.1$, the unsteady effect on free-surface condition is not essential. As the wave elevation is dominant by the near-field disturbance, the wave-like fluctuations can hardly be observed at low forward speed. At moderate Froude number, the unsteady effect becomes to manifest, especially at the gap between two aligned ships $(-0.5<x / L>0.5)$. As the Froude number increases to $F_{n}=0.3$, the difference between 'Steady' and 'Unsteady' can be observed in a wider range of $x / L$, especially at the bow $(x / L=0.5)$ and stern $(x / L=-0.5)$ areas. Fig. $13 a^{-c}$ show the wave elevation components obtained by the present superposition principle. It should be noted that the total wave elevation presented in Fig. 13c is not a simple superposition of the waves produced by two individual hulls without considering the presence of the other one. When we compute the wave elevation produced by $\mathscr{B}_{1}$, the presence of $\mathscr{B}_{2}$ is also considered, treated as an obstacle, by being considered "momentarily" stationary in the body-fixed frame of $\mathscr{B}_{1}$. Therefore, the diffraction and reflection by $\mathscr{B}_{2}$ is considered accounted for and vice versa. These reflected waves can be seen clearly from Fig. 13a and b. 


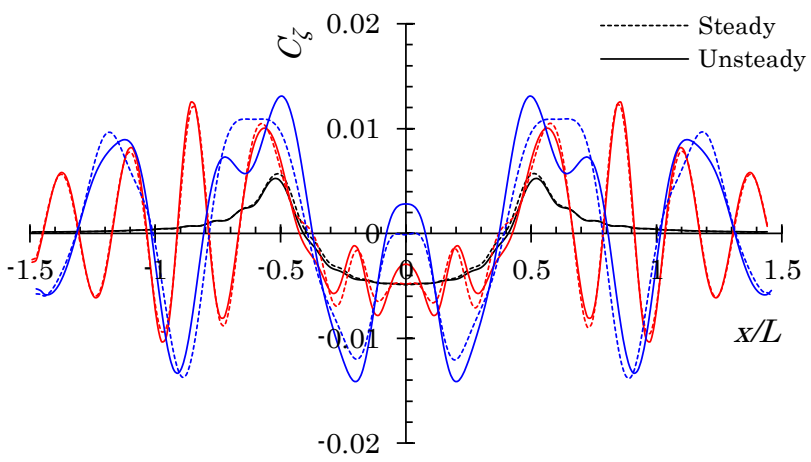

Fig. 12. Wave profiles at the center line between two identical Wigley III hulls in headon encounter with $d_{t} / B=2, d_{l} / L=0$ and $F_{n}=0.3$. The black, red and blue curves correspond to $F_{n}=0.1,0.2$ and 0.3 , respectively.
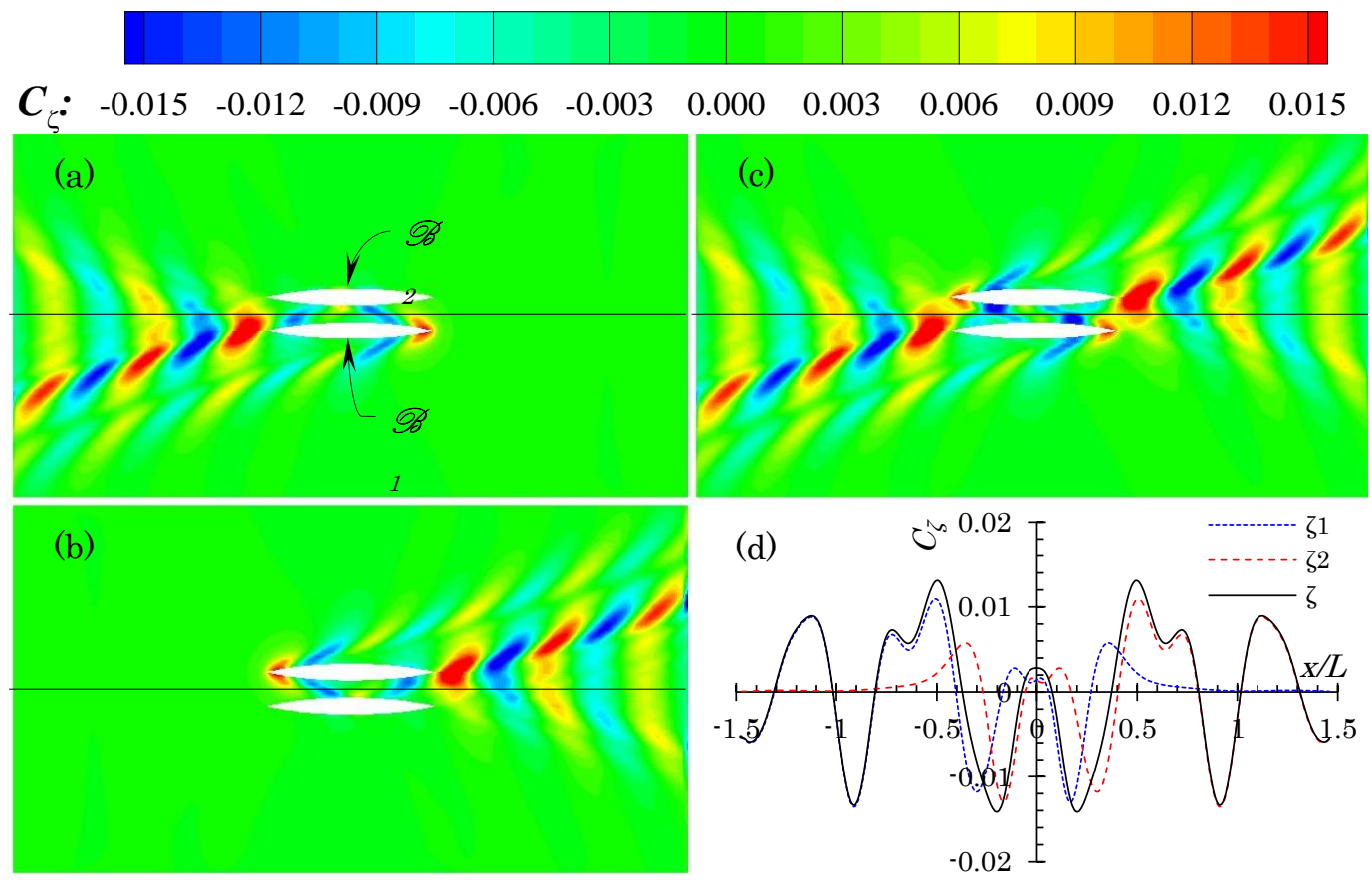

Fig. 13. Waves produced by two Wigley III hulls in head-on encounter with $d_{t} / B=2$, $d_{F}=0$ and $F_{n}=0.3$. (a) $C_{\zeta 1}$, the waves produced by $\mathscr{B}_{1}$ moving at $F_{n}=0.3$ while $\mathscr{B}_{2}$ is momentarily stationary in the body-fixed frame of $\mathscr{B}_{1}$; (b) $C_{\zeta 2}$, the waves produced by $\mathcal{B}_{2}$ moving at $U_{2}$ while $\mathscr{B}_{1}$ is momentarily stationary in the body-fixed frame of $\mathscr{B}_{\dot{2}}$ (c) $C_{\xi}$, the total waves superposing $C_{\zeta 1}$ and $C_{\zeta 2}$ (d) Wave profile at the centreline between two hulls shown in (a), (b) and (c). $x$ in the abscissa of (d) refers to the midship-to-midship distance between left-moving ship ("1") and the encountered ship ("2").

\subsection{The effect of divergent and transverse waves}

Fig. 14 shows the encountering process of two ships in the body-fixed frame of $\mathcal{B}_{2}$. The contour only shows the wave patterns generated by $\mathcal{B}_{2}$ at $F_{n}=0.3$ in isolation in an open domain. For a typical 3D ship, its far-field wave pattern includes two wave systems: bow wave and stern wave. Each wave system has two wave components: divergent wave and transverse wave. In the body-fixed frame of $\mathscr{B}_{2}, \mathscr{B}_{1}$ approaches $\mathcal{B}_{2}$ from its upstream side to its downstream side. Ideally, $\mathcal{B}_{2}$ will experience 6 stages of interference over the entire encountering process:

598 (i): non-interference, onto (ii): local wave disturbance, onto (iii): divergent bow- 
wave disturbance, onto (iv): transverse bow-wave disturbance, onto (v): divergent stern-wave disturbance, onto (vi): transverse wave disturbance. The noninterference stage can only be observed when two ships are sufficient far apart from each other. The transverse bow-waves always interfered with the divergent stern-waves. The disturbance in stage (iii), (iv) and (v) is supposed to be substantial and unpredictable. In the present study, stage (iii), (iv), and (v) are categorized as a combined stage, namely of divergent disturbances. In total, the interference can be divided into three regions: $\mathrm{I}: t<t_{1}, \mathscr{B}_{1}$ is in the local wave disturbance region of $\mathscr{B}_{2} ;$ II: $t_{1}<t<t_{2}, \mathscr{B}_{1}$ is in the divergent wave disturbance region of $\mathscr{B}_{2} ;$ and III: $\mathscr{B}_{1}$ is in the transverse wave disturbance region of $\mathscr{B}_{2}$. Here $t_{1}$ refers to the moment when the bow of $\mathscr{B}_{1}$ reaches the Kelvin envelope of the waves generated by $\mathscr{B}_{2}$, and $t_{2}$ refers to the moment when the stern of $\mathscr{B}_{1}$ leaves the divergent stern`waves generated by $\mathscr{B}_{2}$.

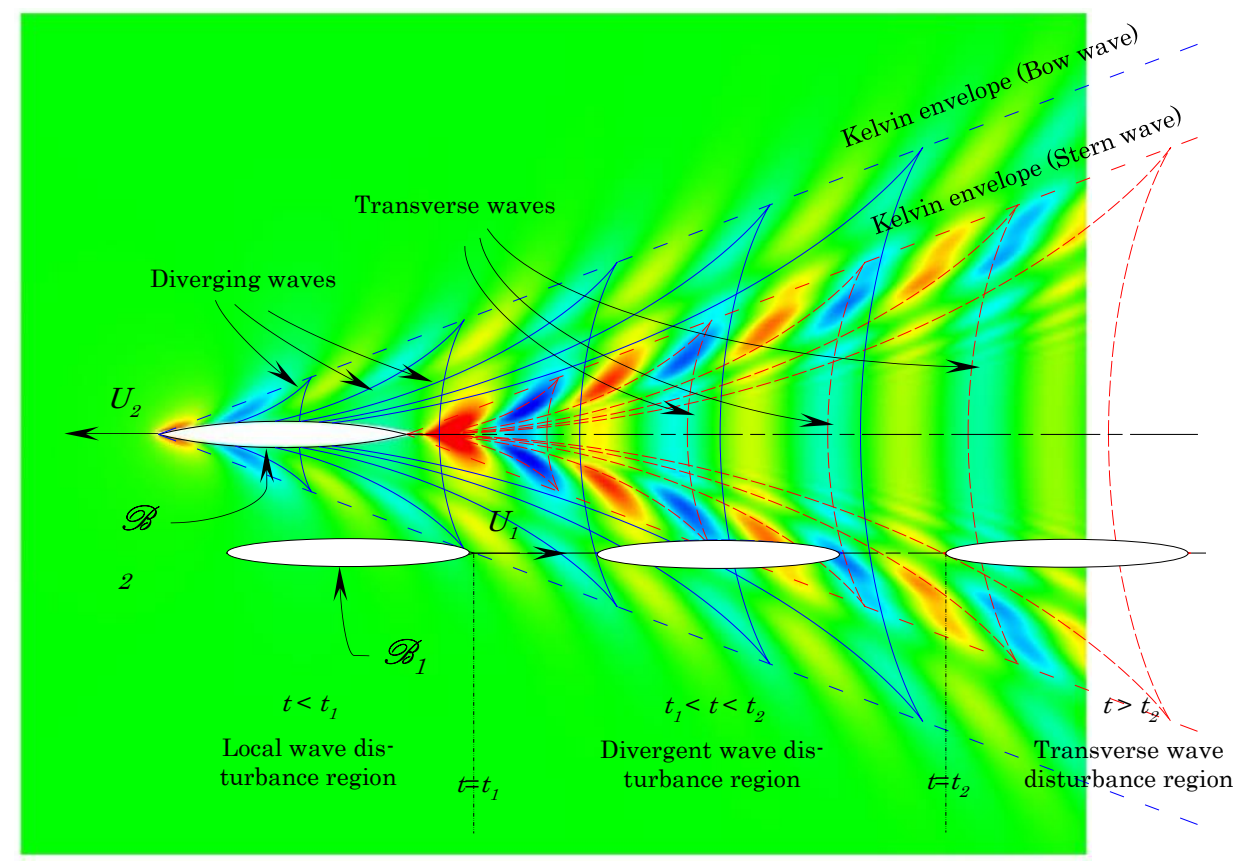

Fig. 14. Sketch showing the encountering process of two ships in the body-fixed frame of $\mathscr{B}_{2}$. The bow and stern of the ships act like two sources (or sinks). The blue and red 617 curves represent bow and stern wave patterns respectively.

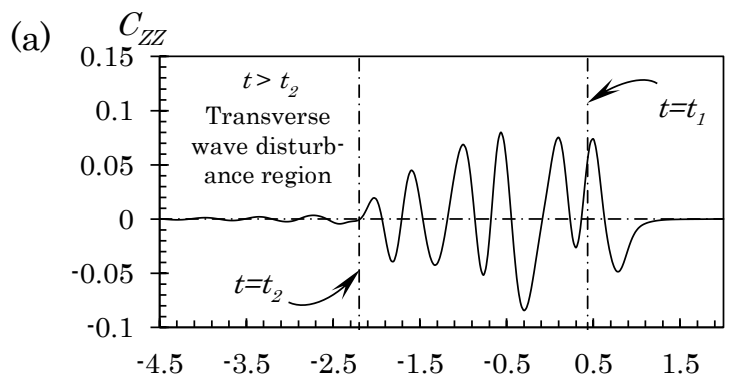


(b)
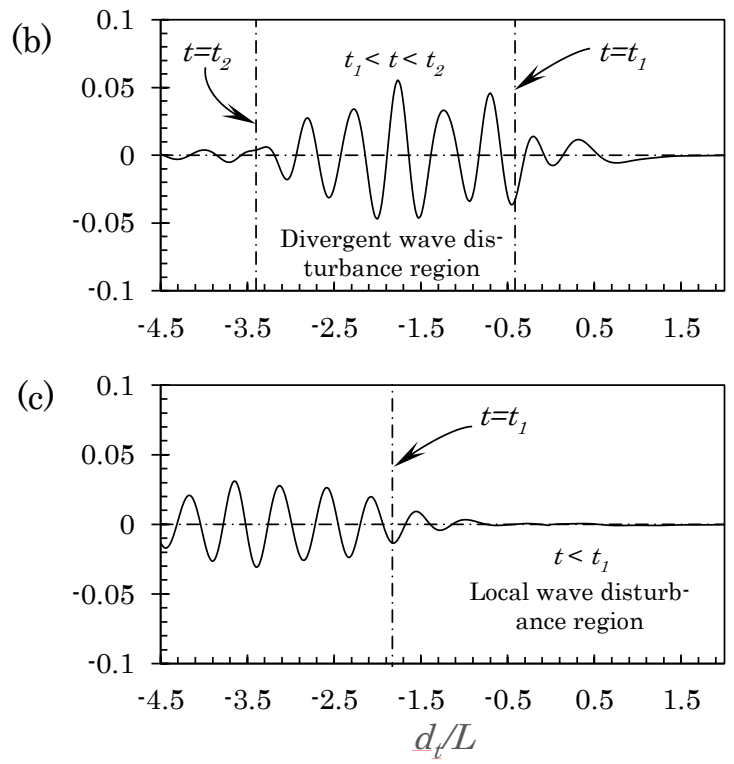

618 Fig. 15. Yaw moment acting on two identical Wigley III hulls in head-on encounter at $619 F_{n=0.3}$. (a) $d_{t} / B=2$; (b) $d_{t} / B=5$; (c) $d_{t} / B=10$. $C_{Z Z}$ is non-dimensionalized by $\frac{1}{2} \rho B T L\left|U_{1} U_{2}\right|$. $620 d / / L>0$ corresponds to $t<t_{s}, d / / L<0$ corresponds to $t>t_{s}$.

Fig. 15 shows the yaw moment on $\mathscr{B}_{1}$ during the aforementioned encountering process. Different lateral separations are investigated here as well. As the lateral separation increases, the non-interference region expands and the disturbance region shifts downstream with regards to the body-fixed frame of $\mathscr{B}_{2}$. It agrees with the physical observation of the far-field waves (Kelvin waves) that confines within the Kelvin wedge downstream. Before $\mathscr{B}_{1}$ reaches the Kelvin envelope, some interactions are observed at $t<t_{1}$, which is due to the disturbance caused by the local waves. To see the synchornization, typical wave patterns at $t<t_{1}$ is shown in Fig. 16a. At $t=t_{1}$, when the bow of $\mathscr{B}_{1}$ meets the divergent waves produced by $\mathcal{B}_{2}$, a very large yaw moment can be induced. When $\mathscr{B}_{1}$ is partly or completely in the divergent disturbance region $\left(t_{1}\right.$ $<t<t_{2}$ ), the interaction becomes significant. The bow and stern waves of $\mathscr{B}_{2}$ interfere in this region, and the wave energy concentrated in this region is usually high, especially when the ship speed is moderate to high. The typical wave pattern at $t_{1}<t<t_{2}$ is shown in Fig. 16b. When $\mathscr{B}_{1}$ completely leaves the divergent disturbance region and enters into the transverse disturbance region $\left(t>t_{2}\right)$, the amplitude of the interaction force decreases with the decay of the transverse waves. The typical wave patterns at $t>t_{2}$ is shown in Fig. 16c. It should be noted that at $d_{t} / B=10$, the forces at the moment $t=t_{2}$ is not captured in Fig. 15c. As the lateral separation increases, $t_{2}$ will shift further downstream.

641 Numerically, to simulate the case with larger lateral separation, the 642 computational domain must be expanded not only laterally, but also to the 643 downstream direction. Much more computational efforts are required to 644 simulate the entire encountering process when the lateral separation becomes 645 large. It can also be seen from Fig. 15 that as the lateral separation increases, 646 the interaction diminishes, but not significantly. The maximum yaw moment at $647 d_{t} / B=10$ still accounts for $40 \%$ of that at $d_{t} / B=2$. It indicates that the hydrody648 namic interaction induced by the far-field waves is quite important at moderate 649 or high speed encountering operation, even though the lateral separation 
653

654

655

656

657

658

659

\section{0}

661

662

663

664

665

666

667

668

669

670

671

672

673

674

675

676

677

678

679

680
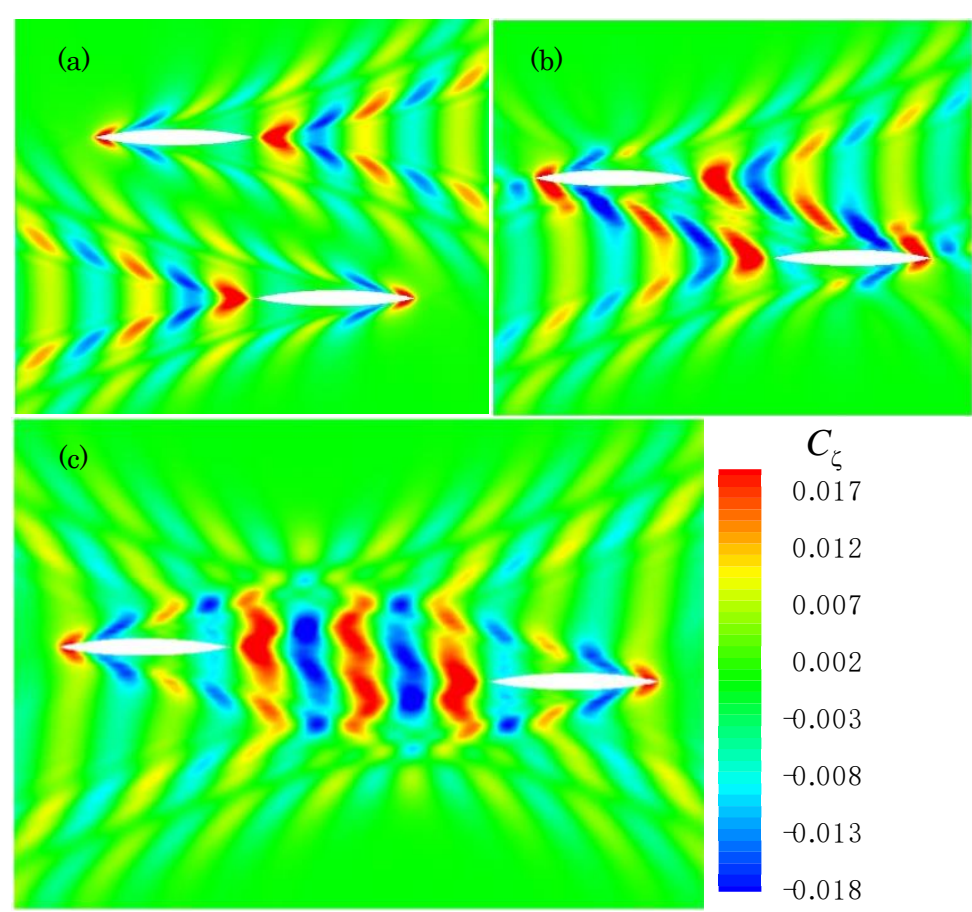

Fig. 16. Wave patterns produced by two identical Wigley III hulls in head-on encounter at $F_{n}=0.3$. (a) $d_{t} / B=10$ and $d_{l} / L=-1$, corresponding to $t<t_{1}$ when a ship is in the other ship's local wave disturbance region; (b) $d_{t} / B=5$ and $d_{l} / L=-1.5$, corresponding to $t_{1}<t$ $<t_{2}$ when a ship is in the other ship's divergent wave disturbance region; (a) $d_{t} / B=2$ and $d l / L=-2.5$, corresponding to $t>t_{2}$ when a ship is in the other ship's transverse wave disturbance region.

\section{CONCLUSIONS}

A linearized free-surface boundary condition was formulated and used to solve the BVP involved in $N$ ship hulls, each moving at its own speeds. Based on superposition principal, the traditional fully-coupled BVP could be decoupled into $N$ sets of independent unsteady BVPs, which can be solved individually in the time domain. The advantage of this decoupled method is that the free-surface boundary condition can be taken into consideration for each set of independent BVPs. Thus, the unsteady hydrodynamic interaction problem can be solved in a fully unsteady manner, and the far-field wave effect can be accounted for.

The present formulation provides an effective way to predict the free-surface effects, with particular application for calculating the lateral interaction force on arbitrary number of ships, each with its own speed. By integrating the present superposition method into a Rankine source (simple-source) panel code, we calculated the unsteady hydrodynamic interaction forces and wave elevation when two ships were under passing, overtaking, or encountering operations. Experimental measurements confirm the applicability of the present approach. Numerical results indicate that the near-field disturbances are the most important component of the interaction force when the encountering speed is low. As the encountering speed increases, the interaction force induced by the farfield waves becomes to manifest gradually. It was found the free-surface effects must be considered at Froude number $F_{n}>0.2$ for slender ships. For blunt-body 
ships, the lower limit of $F_{n}$ is smaller. When the encountering speed reaches $F_{n}$ $=0.3$, free-surface effects become the dominant component. The interaction force induced by the divergent waves could reach a very large value, which may cause ship accidents, such as grounding, capsizing or collisions. By increasing the separation distance between encountering ships could reduce the interaction amplitude, but not significantly. At high encountering speed, the free-surface must be taken into account even though the lateral separation between ships is large.

The superposition method proposed in the present study is not limited to solving the unsteady interaction problem between ships. It can also be applied to predict the hydrodynamic interactions between competitive swimmers in a swimming pool, or between aquatic animals swimming near the free surface. The present approach provides a rational and rapid (real-time capability) tool for analyzing and computing interaction effects, without expending lengthy and detailed-type CFD computations. This can be prohibitively slow to effectively effectively model unsteady multi-body interaction.

\section{ACKNOWLEDGEMENTS}

The first author acknowledges the financially support by a Sir David Anderson Award for his visit at UC Berkeley during which this work was formulated. The third author acknowledges partial support of the American Bureau of Shipping via an endowed chair in Ocean Engineering at UC Berkeley. Discussions with Dr. Lu Wang and Dr. Dongchi Yu of UC Berkeley during the course of this work are appreciated.

\section{REFERENCES}

Bai, K.J., Yeung, R.W., 1974. Numerical solutions to free surface flow problems, Proceedings of the 10th Symposium on Naval Hydrodynamics, Cambridge, Massachusetts, USA.

Bunnik, T., 1999. Seakeeping calculations for ships, taking into account the non-linear steady waves, PhD thesis. Delft University of Technology, The Netherlands.

Collatz, G., 1963. Potentialtheoretische Untersuchung der hydrodynamischen Wechselwirkung zweier Schiffskörper. Jahrbuch der Schiffbautechnischen Gesellschaft 57, 281-389.

Dand, I., 1975. Some aspects of tug-ship interaction, 4th Int. Tug Conv.

Hess, J.L., Smith, A.M.O., 1964. Calculation of nonlifting potential flow about arbitrary threedimensional bodies. Journal of Ship Research 8 (2), 22-44.

Jin, Y., Chai, S., Duffy, J., Chin, C., Bose, N., Templeton, C., 2016. RANS prediction of FLNG-LNG hydrodynamic interactions in steady current. Applied Ocean Research 60, 141-154.

Journee, J.M.J., 1992. Experiments and calculations on 4 Wigley hull forms in head waves, Report No. 0909. Ship Hydromechanics Laboratory, Delft University of Technology, The Netherlands.

Kijima, K., Yasukawa, H., 1985. Manoeuverability of ships in narrow waterway. Journal of the Society of Naval Architects of Japan 23, 25-37.

Kim, Y., Yue, D.K.P., Connell, B.S.H., 2005. Numerical dispersion and damping on steady waves with forward speed. Applied Ocean Research 27 (2), 107-125.

Korsmeyer, F.T., Lee, C.-H., Newman, J., N., 1993. Computation of Ship Interaction Forces in Restricted Waters. Journal of Ship Research 37 (4), 298-306. 
Lataire, E., Vantorre, M., Delefortrie, G., Candries, M., 2012. Mathematical modelling of forces acting on ships during lightering operations. Ocean Engineering 55, 101-115.

Mousaviraad, S.M., Sadat-Hosseini, S.H., Carrica, P.M., Stern, F., 2016a. Ship-ship interactions in calm water and waves. Part 2: URANS validation in replenishment and overtaking conditions. Ocean Engineering 111, 627-638.

Mousaviraad, S.M., Sadat-Hosseini, S.H., Stern, F., 2016b. Ship-ship interactions in calm water and waves. Part 1: Analysis of the experimental data. Ocean Engineering 111, 615-626.

Oltmann, V.P., 1970. Experimentelle Untersuchung der hydrodynamischen Wechselwirkung schiffsähnlicher Körper. Schiff und Hafen 22, 701-707.

Pinkster, J.A., 2004. The influence of a free surface on passing ship effects. International Shipbuilding Progress 51 (4), 313-338.

Shahjada Tarafder, M., Suzuki, K., 2007. Computation of wave-making resistance of a catamaran in deep water using a potential-based panel method. Ocean Engineering 34 (13), 1892-1900.

Sian, A.Y., Maimun, A., Ahmed, Y., 2016. Simultaneous ship-to-ship interaction and bank effect on a vessel in restricted water, Proceedings, 4th MASHCON, Hamburg, German.

Söding, H., Conrad, F., 2005. Analysis of overtaking manoeuvres in a narrow waterway. Ship Technology Research 52, 189-193.

Tuck, E.O., 1966. Shallow water flows past slender bodies. Journal of Fluid Mechanics 26, 81-95.

Tuck, E.O., Newman, J.N., 1974. Hydrodynamic interactions between ships, Proceedings of 10th Symposium on Naval Hydrodynamics, Cambridge, MA, USA, pp. 35-70.

Vantorre, M., Verzhbitskaya, E., Laforce, E., 2002. Model test based formulations of ship-ship interaction forces. Ship Technology Research 49, 124-141.

Varyani, K.S., McGregor, R., Wold, P., 1998. Interactive forces and moments between several ships meeting in conPned waters. Control Engineering Practice 6, 635-642.

Varyani, K.S., McGregor, R., Wold, P., 2002. Identification of trends in extremes of sway-yaw interference for several ships meeting in restricted waters. Ship Technology Research 49, 174-191.

Xiang, X., Faltinsen, O.M., 2010. Maneuvering of Two Interacting Ships in Calm Water, Proceedings, 11th International Symposium on Practical Design of Ships and Other Floating Structures, Rio de Janeiro, RJ, Brazil.

Xu, H., Zou, Z., Zou, L., Liu, X., 2016. Unsteady hydrodynamic interaction between two cylindroids in shallow water based on high-order panel method. Engineering Analysis with Boundary Elements 70, 134-146.

Yeung, R.W., 1975. Surface Waves due to a Maneuvering Air-Cushion Vehicle. Journal of Ship Reaearch 19 (4), 581-607.

Yeung, R.W., 1978. On the interactions of slender ships in shallow water. Journal of Fluid Mechanics $85,143-159$.

Yeung, R.W., Tan, W.T., 1980. Hydrodynamic interactions of ships with fixed obstacles. Journal of Ship Research 24 (1), 50-59.

Yuan, Z.-M., Incecik, A., Ji, C., 2014a. Numerical study on a hybrid mooring system with clump weights and buoys. Ocean Engineering 88 (0), 1-11. 
Yuan, Z.-M., Incecik, A., Jia, L., 2014b. A new radiation condition for ships travelling with very low forward speed. Ocean Engineering 88, 298-309.

Yuan, Z.M., He, S., Paula, K., Incecik, A., Turan, O., Boulougouris, E., 2015. Ship-to-Ship Interaction during Overtaking Operation in Shallow Water. Journal of Ship Research 59 (3), 172-187.

Yuan, Z.M., Incecik, A., 2016a. Investigation of ship-bank, ship-bottom and ship-ship interactions by using potential flow method, Proceedings, 4th International Conference on Ship Manoeuvring in Shallow and Confined Water, Hamburg, Germany.

Yuan, Z.M., Incecik, A., 2016b. Investigation of side wall and ship model interaction, Proceedings, International Conference of Marine Technology (ICMT-2016), September, 2016, Harbin, China.

Zhou, X., Sutulo, S., Guedes Soares, C., 2012. Computation of ship hydrodynamic interaction forces in restricted waters using potential theory. Journal of Marine Science and Application 11 (3), 265275.

Zou, L., Larsson, L., 2013. Numerical predictions of ship-to-ship interaction in shallow water. Ocean Engineering 72, 386-402. 


\section{APPENDIX}

\section{6-DOF Interaction Forces and Moment Due to The Encountering of Two Wigley- III Hulls of Identical $\boldsymbol{F}_{n}$}

Fig. A. 1-Fig. A. 2 show the effect of encountering speed and lateral separation on the interaction forces in 6 Degrees of Freedom. When the lateral clearance between two ships is small $\left(d_{t} / B=2\right)$, both near-field and far-field disturbance can be observed. However, only far-field wave disturbance can be overserved at high speed encountering when the lateral clearance becomes large $\left(d_{t} / B=10\right)$.

(a)

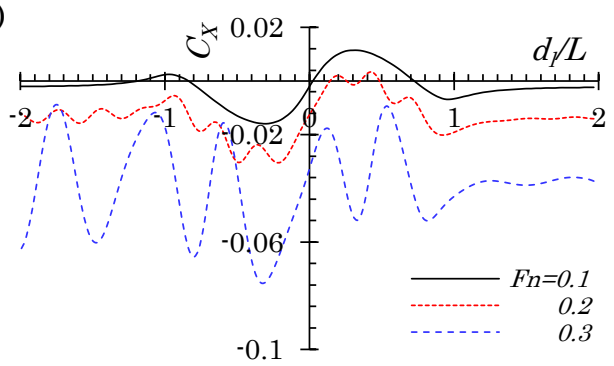

(c) ${ }_{-2}$

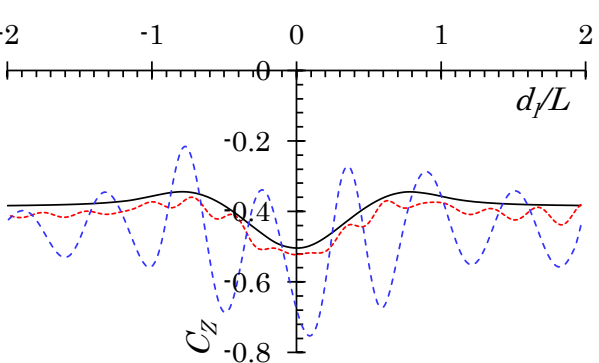

(e)

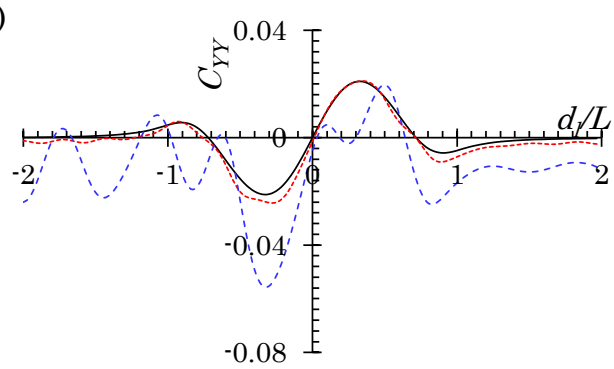

(b)

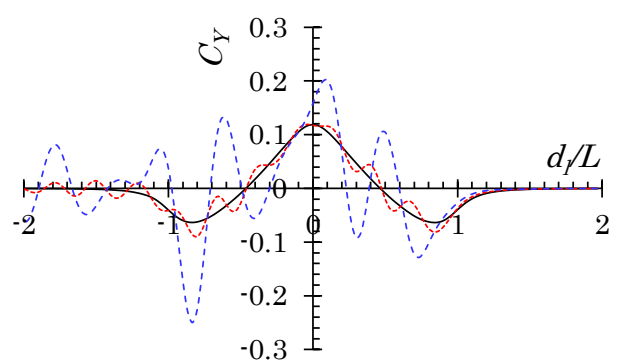

(d)

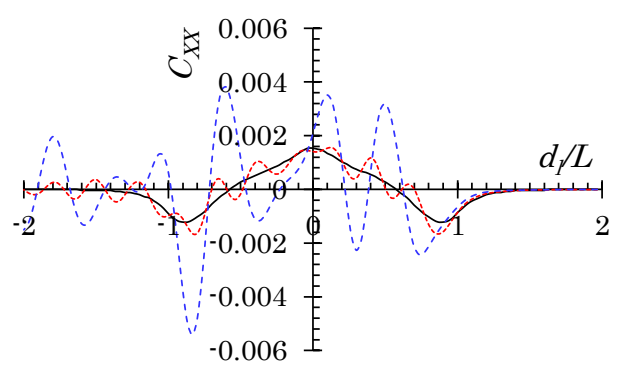

(f)

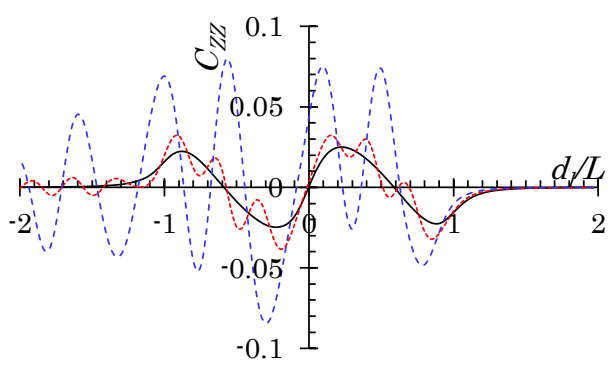

Fig. A. 1. Forces and moments acting on two identical Wigley III hulls in head-on encounter with $d_{t} / B=2$. (a) Surge force; (b) sway force; (c) heave force; (d) roll moment; (e) pitch moment; (f) yaw moment. Forces are non-dimensionalized by $\frac{1}{2} \rho \boldsymbol{B} \boldsymbol{T}\left|\boldsymbol{U}_{\mathbf{1}} \boldsymbol{U}_{\mathbf{2}}\right|$ and moments are non-dimensionalized by $\frac{1}{2} \rho \boldsymbol{B} \boldsymbol{T} \boldsymbol{L}\left|\boldsymbol{U}_{1} \boldsymbol{U}_{2}\right|$. 
(a)

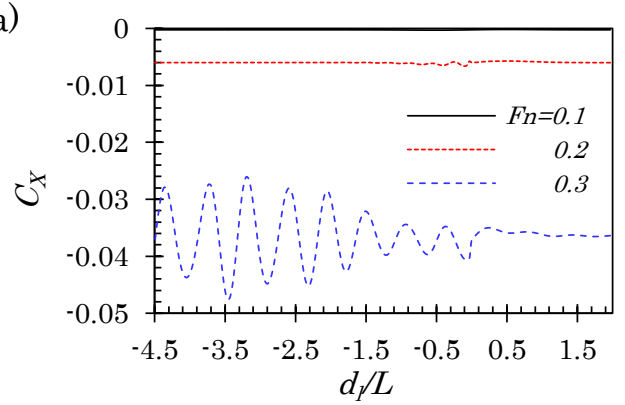

(c)

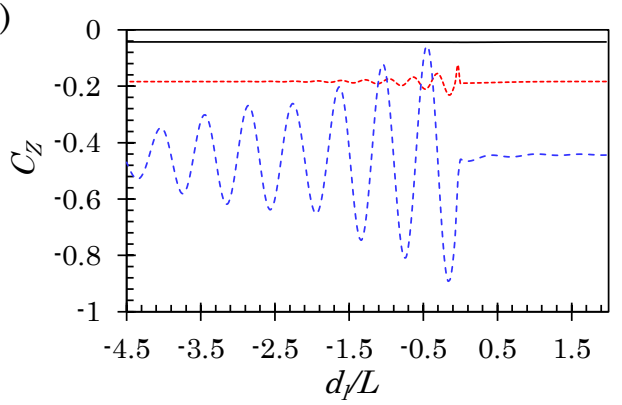

(e)

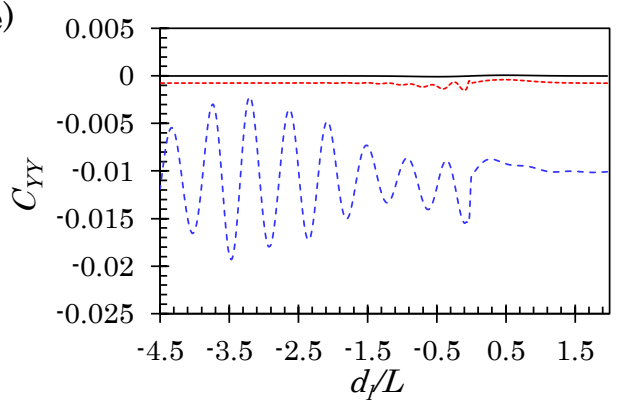

(b)

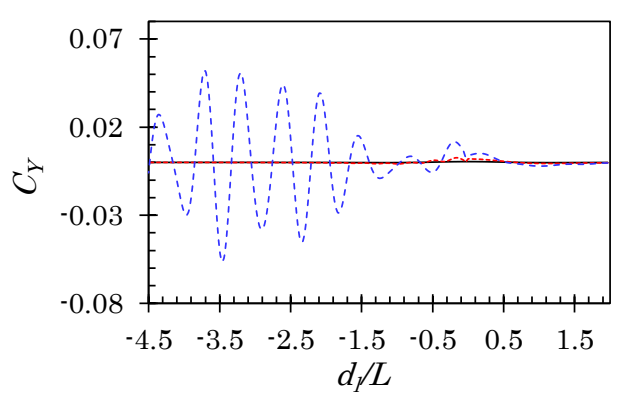

(d)

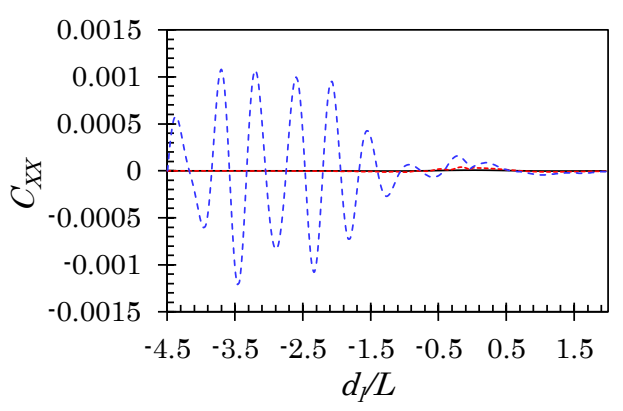

(f)

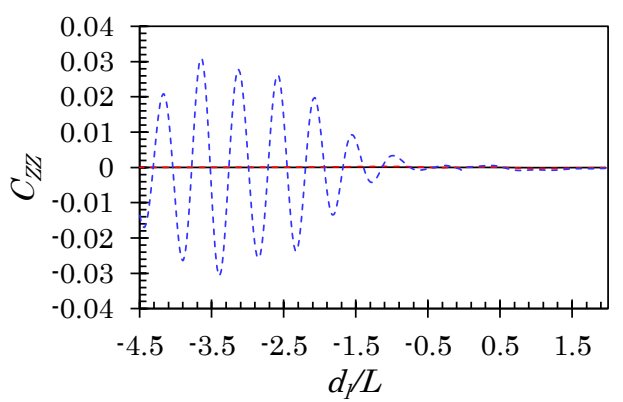

Fig. A. 2 Forces and moments acting on two identical Wigley III hulls in head-on encounter with $d t / B=10$. (a) Surge force; (b) sway force; (c) heave force; (d) roll moment; (e) pitch moment; (f) yaw moment. 